\title{
One-parameter automorphism groups of operator algebras allowing spectral projections
}

\author{
GEORGE A. ELLIOTT AND LÁSZLÓ ZSIDÓ \\ Mathematics Institute, Universitetsparken 5, DK-2100 Copenhagen $\varnothing$, Denmark; \\ Mathematisches Institut A, Pfaffenwaldring 57, D-7000 Stuttgart 80, West Germany
}

(Received 11 May 1982)

\begin{abstract}
The property of a one-parameter $C^{*}$ - (or $W^{*}$-) dynamical system that the spectral subspaces corresponding to the three subsets $(-\infty, 0),\{0\}$, and $(0,+\infty)$ add up to the whole algebra is reformulated. If the $C^{*}$-algebra is prime (or the $W^{*}$ algebra is a factor), an equivalent property is that the spectrum is finite.
\end{abstract}

\section{Introduction}

One of the classical examples of a non-complemented closed linear subspace of a Banach space is the following (see e.g. [10]):

Let $\mathbb{T}=\{\zeta \in \mathbb{C} ;|\zeta|=1\}, C(\mathbb{T})=$ the Banach space of all complex continuous functions on $\mathbb{T}$, and $A(\mathbb{T})=$ the set of all $f \in C(\mathbb{T})$ allowing a continuous extension to the disk $\{\zeta \in \mathbb{C} ;|\zeta| \leq 1\}$ which is analytic on the interior. Then $A(\mathbb{T})$ is a closed linear subspace of $C(\mathbb{T})$ without topological complement. $C(\mathbb{T})$ is also a $C^{*}$-algebra, and, if one defines the strongly continuous one-parameter group $\tau$ of $*$-automorphisms of $C(\mathbb{T})$ by

$$
\left(\tau_{t}(f)\right)(\zeta)=f\left(e^{-i t} \zeta\right)
$$

then $A(\pi)$ is exactly the spectral subspace $C(\pi)^{\tau}((-\infty, 0])$ of $\tau$ (see [1]). Thus, the above example suggests that if $(A, \alpha)$ is a one-parameter $C^{*}$-dynamical system (see $[12])$, then the spectral subspace $A^{\alpha}((-\infty, 0])$ seldom has a topological complement.

The purpose of this paper is to give a complete description of all one-parameter $C^{*}$-dynamical systems $(A, \alpha)$, resp. $W^{*}$-dynamical systems $(M, \alpha)$, for which $A^{\alpha}((-\infty, 0])$, resp. $M^{\alpha}((-\infty, 0])$, has a topological complement. Using the techniques developed in [5], we prove that a necessary and sufficient condition for this to hold, at least, in the case of a $C^{*}$-dynamical system, after passing to an invariant essential closed two-sided ideal, is that, locally, in a certain sense, $\alpha$ has finite spectrum, and, moreover, the cardinality of the local spectrum of $\alpha$ is uniformly bounded ( $\S 3$ ). In the case that $A$ is prime, resp. $M$ is a factor, the spectral subspace of $\alpha$ corresponding to $(-\infty, 0]$ has a topological complement if and only if the spectrum of $\alpha$ is finite $(\S 2)$. 


\section{Preliminaries}

In this paragraph we recall some general facts concerning one-parameter groups of operators.

We will call a dual pair of Banach spaces any pair $(X, \mathscr{F})$ of complex Banach spaces, together with a bilinear functional $X \times \mathscr{F} \ni(x, \phi) \mapsto\langle x, \phi\rangle \in \mathbb{C}$, such that:

(i) $\|x\|=\sup _{\|\phi\| \leq 1}|\langle x, \phi\rangle|$ for any $x \in X$;

(ii) $\|\phi\|=\sup _{\|x\| \leq 1}|\langle x, \phi\rangle|$ for any $\phi \in \mathscr{F}$;

(iii) the convex hull of any relatively $\sigma(X, \mathscr{F})$-compact subset of $X$ is relatively $\sigma(X, \mathscr{F})$-compact;

(iv) the convex hull of any relatively $\sigma(\mathscr{F}, X)$-compact subset of $\mathscr{F}$ is relatively $\sigma(\mathscr{F}, X)$-compact.

If $X$ is a complex Banach space and $X^{*}$ its dual, then the pairs $\left(X, X^{*}\right)$ and $\left(X^{*}, X\right)$, endowed with the natural pairing between $X$ and $X^{*}$, are dual pairs of Banach spaces.

If $(X, \mathscr{F})$ is a dual pair of Banach spaces, then the uniform boundedness principle holds in $X$ with respect to $\sigma(X, \mathscr{F})([9$, th. 2.8 .6$])$; moreover, quite general $X$-valued maps, defined on a locally compact space endowed with a Radon measure, are $\sigma(X, \mathscr{F})$-integrable ([1, prop. 1.2]; [2, prop. 1.4]). Let us denote by $B_{\mathscr{F}}(X)$ the Banach algebra of all $\sigma(X, \mathscr{F})$-continuous linear operators on $X$. For each $T \in B_{\mathscr{F}}(X)$ the adjoint $T^{\mathscr{F}} \in B_{X}(\mathscr{F})$ of $T$ in $\mathscr{F}$ is defined by

$$
T^{\mathscr{F}}(\phi)=\phi \circ T, \quad \phi \in \mathscr{F} \text {. }
$$

In the cases $\mathscr{F}=X^{*}$ and $X=\mathscr{F}^{*}$ we use the usual notation $T^{*}$ for $T^{\mathscr{F}}$.

Let $(X, \mathscr{F})$ be a dual pair of Banach spaces. A one-parameter group $U$ in $B_{\mathscr{F}}(X)$ is a mapping $U: \mathbb{R} \rightarrow B_{\mathscr{F}}(X)$ such that

$$
\begin{aligned}
U_{0} & =\text { identity map of } X, \\
U_{t_{1}+t_{2}} & =U_{t_{1}} U_{t_{2}}, \quad t_{1}, t_{2} \in \mathbb{R} .
\end{aligned}
$$

$U$ is called $\sigma(X, \mathscr{F})$-continuous if for each $x \in X$ the mapping

$$
\mathbb{R} \ni t \mapsto U_{t}(x) \in X
$$

is $\sigma(X, \mathscr{F})$-continuous. In this case one can define the dual group $U^{\mathscr{F}}$ in $B_{X}(\mathscr{F})$ by

$$
U_{t}^{\mathscr{F}}=\left(U_{t}\right)^{\mathscr{F}}, \quad t \in \mathbb{R},
$$

and $U^{\mathscr{F}}$ is $\sigma(\mathscr{F}, X)$-continuous. We note that if $\mathscr{F}=X^{*}$ then a $\sigma(X, \mathscr{F})$-continuous one-parameter group $U$ in $B(X)=B_{\mathscr{F}}(X)$ is always strongly continuous. In this case we use the notation $U^{*}$ for $U^{\mathscr{F}}$.

For bounded, or even certain non-quasianalytic $\sigma(X, \mathscr{F})$-continuous one-parameter groups in $B_{\mathscr{F}}(X)$ a valid theory of spectral subspaces can be developed ([1], [11], [4], [3]). Since we have $C^{*}$ - and $W^{*}$-dynamical systems in view, we shall deal in the sequel only with one-parameter groups of isometries in $B_{\mathscr{F}}(X)$ :

Let $(X, \mathscr{F})$ be a dual pair of Banach spaces and $U$ a $\sigma(X, \mathscr{F})$-continuous one-parameter group of isometries in $B_{\mathscr{F}}(X)$. For each $f \in L^{1}(\mathbb{R})$ one can define $U_{f} \in B_{\mathscr{F}}(X)$ by

$$
\left\langle U_{f}(x), \phi\right\rangle=\int_{-\infty}^{+\infty} f(t)\left\langle U_{t}(x), \phi\right\rangle d t, \quad x \in X, \phi \in \mathscr{F} .
$$


With $\hat{f}$ denoting the inverse Fourier transform of $f$, defined by

$$
\hat{f}(s)=\int_{-\infty}^{+\infty} f(t) e^{i s t} d t, \quad s \in \mathbb{R},
$$

the spectral subspace of $U$, associated to a closed set $F \subset \mathbb{R}$, is given by

$$
X^{U}(F)=\left\{x \in X ; U_{f}(x)=0 \quad \text { for all } f \in L^{1}(\mathbb{R}) \text { with } F \cap \operatorname{supp}(\hat{f})=\varnothing\right\},
$$

([1]). Denoting by $U_{z}$ the analytic extension of $U$ in $z \in \mathbb{C}([2])$ one has for each $\lambda \in \mathbb{R}$,

$$
\begin{aligned}
X^{U}((-\infty, \lambda]) & =\left\{x \in \bigcap_{k=0}^{\infty} \mathscr{D}_{U_{-k i}} ; \varlimsup_{k \rightarrow \infty}\left\|U_{-k i}(x)\right\|^{1 / k} \leq e^{\lambda}\right\} \\
& =\left\{x \in \bigcap_{\operatorname{Im} z \leq 0} \mathscr{D}_{U_{z}} ;\left\|U_{z}(x)\right\| \leq e^{\lambda|\operatorname{Im} z|}\|x\| \quad \text { for } \operatorname{Im} z \leq 0\right\}, \\
X^{U}([\lambda,+\infty))= & \left\{x \in \bigcap_{k=0}^{\infty} \mathscr{D}_{U_{k i}} ; \varlimsup_{k \rightarrow \infty}\left\|U_{k i}(x)\right\|^{1 / k} \leq e^{-\lambda}\right\} \\
= & \left\{x \in \bigcap_{\operatorname{Im} z \geq 0} \mathscr{D}_{U_{z}} ;\left\|U_{z}(x)\right\| \leq e^{-\lambda|\operatorname{Im} z|}\|x\| \quad \text { for } \operatorname{Im} z \geq 0\right\}
\end{aligned}
$$

([16]). The spectrum of $U$ is the closed set

$$
\begin{aligned}
\sigma(U) & =\left\{\lambda \in \mathbb{R} ; \begin{array}{l}
\text { for each neighbourhood } N \text { of } \lambda \text { there is } \\
f_{N} \in L^{1}(\mathbb{R}) \text { with } \operatorname{supp}\left(\hat{f}_{N}\right) \subset N \text { and } U_{f_{N}} \neq 0
\end{array}\right\} \\
& =\left\{\lambda \in \mathbb{R} ; \text { for all } \lambda_{1}, \lambda_{2} \in \mathbb{R} \text { with } \lambda_{1}<\lambda<\lambda_{2}, X^{U}\left(\left[\lambda_{1}, \lambda_{2}\right]\right) \neq 0\right\}
\end{aligned}
$$

([1]). We assume familiarity in handling spectral subspaces and spectra.

(1.1) Theorem. Let $(X, \mathscr{F})$ be a dual pair of Banach spaces and $U$ a $\sigma(X, \mathscr{F})$ continuous one-parameter group of isometries in $B_{\mathscr{F}}(X)$. If $\lambda$ is an isolated point of $\sigma(U)$ then there is a (unique) projection $P_{\lambda}^{U} \in B_{\mathscr{F}}(X)$ with

$$
\text { image of } P_{\lambda}^{U}=X^{U}(\{\lambda\}) \text {; }
$$

kernel of $P_{\lambda}^{U}=X^{U}(\sigma(U) \backslash\{\lambda\})$;

$P_{\lambda}^{U} U_{t}=U_{t} P_{\lambda}^{U}, t \in \mathbb{R} ;$ and

$$
\left\|P_{\lambda}^{U}\right\|=1 \text {. }
$$

Proof. Replacing $U$ by $\mathbb{R} \ni t \mapsto e^{-i \lambda t} U_{t} \in B_{\mathscr{F}}(X)$, we may assume that $\lambda=0$. Choose some $f_{0} \in L^{1}(\mathbb{R})$ such that:

$$
\begin{gathered}
\hat{f}_{0}(s)=1 \quad \text { for } s \text { in a neighbourhood of } 0, \\
\operatorname{supp}\left(\hat{f}_{0}\right) \cap(\sigma(U) \backslash\{0\})=\varnothing,
\end{gathered}
$$

and put $P_{0}^{U}=U_{f_{0}} \in B_{\mathscr{F}}(X)$. It is easy to see that $P_{0}^{U}$ is a projection with image of $P_{0}^{U}=X^{U}(\{0\})$, kernel of $P_{0}^{U}=X^{U}(\sigma(U) \backslash\{0\})$. Obviously,

$$
P_{0}^{U} U_{t}=U_{t} P_{0}^{U}, \quad t \in \mathbb{R} \text {. }
$$

By the arguments from [17, pp. 242, 243] we have

$$
P_{0}^{U}(x)=x=\text { norm- } \lim _{T \rightarrow+\infty} \frac{1}{2 T} \int_{-T}^{T} U_{t}(x) d t,
$$


for $x \in X^{U}(\{0\})$, and

$$
P_{0}^{U}(x)=0=\text { norm- } \lim _{T \rightarrow+\infty} \frac{1}{2 T} \int_{-T}^{T} U_{t}(x) d t,
$$

for $x \in \bigcup_{\mu<0} X^{U}((-\infty, \mu])$ or $\bigcup_{\mu>0} X^{U}([\mu,+\infty))$.

For each $x$ belonging to

$$
\bigcup_{K \in \mathbb{R} \text { compact }} X^{U}(K) \subset X^{U}(\{0\})+\left(\bigcup_{\mu<0} X^{U}((-\infty, \mu])\right)+\left(\bigcup_{\mu>0} X^{U}([\mu,+\infty))\right)
$$

it follows that, successively,

$$
\begin{aligned}
P_{0}^{U}(x) & =\text { norm-lim } \lim _{T \rightarrow+\infty} \frac{1}{2 T} \int_{-T}^{T} U_{t}(x) d t, \\
\left\|P_{0}^{U}(x)\right\| & \leq\|x\| .
\end{aligned}
$$

Since the unit ball of $\bigcup_{K \subset R}$ compact $X^{U}(K)$ is $\sigma(X, \mathscr{F})$-dense in the closed unit ball of $X$ and $P_{0}^{U}$ is $\sigma(X, \mathscr{F})$-continuous, it follows that $\left\|P_{0}^{U}\right\| \leq 1$. On the other hand, since $0 \in \sigma(U)$, we have $P_{0}^{U} \neq 0$ and therefore $\left\|P_{0}^{U}\right\|=1$.

For each set $S$ we denote by $|S|$ the cardinal number of $S$.

(1.2) Corollary. Let $(X, \mathscr{F})$ be a dual pair of Banach spaces and $U$ a $\sigma(X, \mathscr{F})$ continuous one-parameter group of isometries in $B_{\mathscr{F}}(X)$. If $F$ is a finite set of isolated points of $\sigma(U)$ then there is a (unique) projection $P_{F}^{U} \in B_{\mathscr{F}}(X)$ with:

$$
\begin{aligned}
& \text { image of } P_{F}^{U}=X^{U}(F) ; \\
& \text { kernel of } P_{F}^{U}=X^{U}(\sigma(U) \backslash F) ; \\
& P_{F}^{U} U_{t}=U_{t} P_{F}^{U}, t \in \mathbb{R} ; \text { and } \\
& \left\|P_{F}^{U}\right\| \leq|F| .
\end{aligned}
$$

Proof. With the notation of theorem (1.1) put $P_{F}^{U}=\sum_{\lambda \in F} P_{\lambda}^{U}$.

In particular, if $\sigma(U)$ is finite then there exists a bounded linear projection of norm $\leq|\sigma(U)|$ onto each spectral subspace of $U$. The aim of this paper is to prove a converse of this statement for $C^{*}$ - and $W^{*}$-dynamical systems: if $U$ is a $C^{*}$ - or $W^{*}$-dynamical system and there exists a bounded linear projection onto $X^{U}((-\infty, 0])$ then, 'locally', $\sigma(U)$ is finite.

Next we examine in a quite general situation the consequences for $U$ of the existence of a bounded linear projection onto $X^{U}((-\infty, 0])$.

(1.3) Theorem. Let $\mathscr{F}$ be a complex Banach space, $X$ its dual, and $U$ a $\sigma(X, \mathscr{F})$ continuous one-parameter group of isometries in $B_{\mathscr{F}}(X)$. Then there exists a linear mapping

$$
B(X) \ni P \mapsto P^{U} \in B(X)
$$

such that for every $P \in B(X)$

and

$$
\begin{gathered}
\left\|P^{U}\right\| \leq\|P\|, \\
P^{U} U_{t}=U_{t} P^{U}, \quad t \in \mathbb{R}
\end{gathered}
$$

$$
\begin{aligned}
F \subset \mathbb{R} \text { closed, } P(x)=x & \text { for } x \in X^{U}(F) \\
\Rightarrow P^{U}(x)=x & \text { for } x \in X^{U}(F)
\end{aligned}
$$




$$
\begin{aligned}
F \subset \mathbb{R} \text { closed, } P(x)=0 & \text { for } x \in X^{U}(F) \\
\Rightarrow P^{U}(x)=0 & \text { for } x \in X^{U}(F) ;
\end{aligned}
$$

$F \subset \mathbb{R}$ closed, $P(x) \in X^{U}(F)$ for $x \in X$ with $\mathbb{R} \ni t \mapsto U_{t}(x) \in X$ norm-continuous $\Rightarrow P^{U}(x) \in X^{U}(F)$ for $x \in X$ with $\mathbb{R} \ni t \mapsto U_{t}(x) \in X$ norm-continuous.

Proof. For the proof we adapt an argument from [14]. The additive group $\mathbb{R}$ being commutative, there exists an invariant mean $m$ on the vector space of all bounded complex functions on $\mathbb{R}$ (see for example [7, th. 1.2.1]).

Let $P \in B(X)$. For every $x \in X$ and $\phi \in \mathscr{F}$ we define $f_{x, \phi}: \mathbb{R} \rightarrow \mathbb{C}$ by

$$
f_{x, \phi}(t)=\left\langle U_{t} P U_{-t}(x), \phi\right\rangle, \quad t \in \mathbb{R} .
$$

Then

$$
\sup _{t \in \mathbb{R}}\left|f_{x, \phi}(t)\right| \leq\|P\|\|x\|\|\phi\|, \quad x \in X, \phi \in \mathscr{F},
$$

and $x \times \mathscr{F} \ni(x, \phi) \mapsto f_{x, \phi}$ is a bilinear mapping into the vector space of all bounded complex functions on $\mathbb{R}$. It follows that

$$
X \times \mathscr{F} \ni(x, \phi) \mapsto m\left(f_{x, \phi}\right)
$$

is a bounded bilinear functional of norm $\leq\|P\|$. Since $X$ is the dual space of $\mathscr{F}$, there exists $P^{U} \in B(X)$ with

$$
\begin{gathered}
\left\|P^{U}\right\| \leq\|P\|, \\
\left\langle P^{U}(x), \phi\right\rangle=m\left(f_{x, \phi}\right), \quad x \in X, \phi \in \mathscr{F} .
\end{gathered}
$$

Clearly, $P^{U}$ depends linearly on $P$.

Let $s \in \mathbb{R}$ be arbitrary. For every $x \in X$ and $\phi \in \mathscr{F}$ we have

so by the invariance of $m$ we get

$$
f_{U_{s}(x), U_{-s}^{*}(\phi)}(t)=f_{x, \phi}(t-s), \quad t \in \mathbb{R},
$$

$$
\begin{aligned}
\left\langle U_{-s} P^{U} U_{s}(x), \phi\right\rangle & =\left\langle P^{U} U_{s}(x), U_{-s}^{*}(\phi)\right\rangle=m\left(f_{U_{s}(x), U_{-s}^{*}(\phi)}\right) \\
& =m\left(f_{x, \phi}\right)=\left\langle P^{U}(x), \phi\right) .
\end{aligned}
$$

It follows that $U_{-s} P^{U} U_{s}=P^{U}$, and $P^{U} U_{s}=U_{s} P^{U}$.

Let us assume that $F \subset \mathbb{R}$ is a closed set such that $P(x)=x$ for $x \in X^{U}(F)$. Then we get for each $x \in X^{U}(F)$, successively,

$$
\begin{aligned}
f_{x, \phi}(t) & =\langle x, \phi\rangle, & & \phi \in \mathscr{F}, t \in \mathbb{R}, \\
\left\langle P^{U}(x), \phi\right\rangle & =m\left(f_{x, \phi}\right)=\langle x, \phi\rangle, & & \phi \in \mathscr{F}, \\
P^{U}(x) & =x . & &
\end{aligned}
$$

Next let $F \subset \mathbb{R}$ be a closed set such that $P(x)=0$ for $x \in X^{U}(F)$. Then we get for each $x \in X^{U}(F)$, successively,

$$
\begin{aligned}
f_{x, \phi}(t) & =0, & & \phi \in \mathscr{F}, t \in \mathbb{R}, \\
\left\langle P^{U}(x), \phi\right\rangle & =m\left(f_{x, \phi}\right)=0, & & \phi \in \mathscr{F}, \\
P^{U}(x) & =0 . & &
\end{aligned}
$$

Finally, let $F \subset \mathbb{R}$ be a closed set such that $P(x) \in X^{U}(F)$ for $x \in X$ with $\mathbb{R} \ni t \mapsto U_{t}(x)$ norm-continuous. Then we have for each $x \in X$ with $\mathbb{R} \ni t \mapsto U_{t}(x)$ norm-continuous 
and for each $\phi \in \mathscr{F}$ in the annihilator of $X^{U}(F)$,

$$
\begin{gathered}
f_{x, \phi}(t)=0, \quad t \in \mathbb{R}, \\
\left\langle P^{U}(x), \phi\right\rangle=m\left(f_{x, \phi}\right)=0 .
\end{gathered}
$$

By the Hahn-Banach theorem it follows that $P^{U}(x) \in X^{U}(F)$ for $x \in X$ with $\mathbb{R} \ni t \mapsto$ $U_{t}(x)$ norm-continuous.

(1.4) Corollary. Let $\mathscr{F}$ be a complex Banach space, $X$ its dual, and $U$ a $\sigma(X, \mathscr{F})$ continuous one-parameter group of isometries in $B_{\mathscr{F}}(X)$. If there exist $P \in B(X)$ and $\lambda, \mu \in \mathbb{R}, \quad \lambda \leq \mu, \quad$ such that $P(x)=x$ for $x$ in the norm-closure of $\bigcup_{K \in(-\infty, \lambda] \text { compact }} X^{U}(K), P(x) \in X^{U}((-\infty, \mu])$ for $x \in X$ with $\mathbb{R} \ni t \mapsto U_{t}(x) \in X$ norm-continuous, then we have for the spectrum $\sigma\left(U_{-i}\right)$ of the analytic generator $U_{-i}$ of $U$,

$$
\sigma\left(U_{-i}\right) \neq \mathbb{C} \text {. }
$$

Proof. By theorem (1.3) there exists $P^{U} \in B(X)$ such that $P^{U}(x)=x$ for $x$ in the norm-closure of $\bigcup_{K \subset(-\infty, \lambda] \text { compact }} X^{U}(K), P^{U}(x) \in X^{U}((-\infty, \mu])$ for $x \in X$ with $\mathbb{R} \ni t \mapsto U_{t}(x) \in X$ norm-continuous, and $P^{U} U_{t}=U_{t} P^{U}, t \in \mathbb{R}$. Set

$$
Y=\left\{x \in X ; \mathbb{R} \ni t \mapsto U_{t}(x) \in X \text { is norm-continuous }\right\} .
$$

By [4, prop. 2.10], $Y$ is a $U$-invariant norm-closed linear subspace of $X$, the restriction $U \mid Y$ of $U$ to $Y$ is a strongly continuous one-parameter group of isometries in $B(Y)$, and the closed unit ball of $Y$ is $\sigma(X, \mathscr{F})$-dense in the closed unit ball of $X$. Since $P^{U}$ is bounded and commutes with every $U_{t}$, it leaves $Y$ invariant and $P^{U} \mid Y$ commutes with every $(U \mid Y)_{t}=U_{t} \mid Y$.

For each compact $K \subset \mathbb{R}$ we have

$$
Y^{U \mid Y}(K) \subset Y^{U \mid Y}(K \cap(-\infty, \lambda])+Y^{U \mid Y}([\lambda-1,+\infty)),
$$

so

$$
\left(1-P^{U}\right) Y^{U \mid Y}(K) \subset\left(1-P^{U}\right) Y^{U \mid Y}([\lambda-1,+\infty)) \subset Y^{U \mid Y}([\lambda-1,+\infty)) .
$$

Since $\bigcup_{K \subset \mathbb{R} \text { compact }} Y^{U \mid Y}(K)$ is norm-dense in $Y$, it follows that

$$
\left(1-P^{U}\right) Y \subset Y^{U \mid Y}([\lambda-1,+\infty)) \text {. }
$$

Thus

$$
\begin{aligned}
Y & =P^{U}(Y)+\left(1-P^{U}\right)(Y) \\
& \subset Y^{U \mid Y}((-\infty, \mu])+Y^{U \mid Y}([\lambda-1,+\infty)) \\
& \subset \mathscr{D}_{(U \mid Y)_{-i}}+\mathscr{D}_{(U \mid Y)_{i}} .
\end{aligned}
$$

By $\left[18\right.$, cor. 2.4] it follows that $\sigma\left((U \mid Y)_{-i}\right) \subset[0,+\infty)$. In particular, $-1 \notin \sigma\left((U \mid Y)_{-i}\right)$. Set

$$
c=\left\|\left(1+(U \mid Y)_{-i}\right)^{-1}\right\| .
$$

Now let $x \in X$ be arbitrary. Since the closed unit ball of $Y$ is $\sigma(X, \mathscr{F})$-dense in the closed unit ball of $X$, there is a net $\left(y_{i}\right)_{i \in I}$ in $Y$ such that $\left\|y_{i}\right\| \leq\|x\|, i \in I, y_{i} \rightarrow x$ in $\sigma(X, \mathscr{F})$. Writing

$$
z_{i}=\left(1+(U \mid Y)_{-i}\right)^{-1}\left(y_{i}\right), \quad i \in I
$$


we have

$$
\begin{aligned}
\left\|z_{i}\right\| & \leq c\left\|y_{i}\right\| \leq c\|x\|, & & i \in I, \\
y_{i} & =z_{i}+U_{-i}\left(z_{i}\right), & & i \in I .
\end{aligned}
$$

But by the Alaoglu theorem there exists a subnet $\left(z_{i_{\lambda}}\right)_{\lambda \in L}$ of $\left(z_{i}\right)_{i \in I}$ which converges in the $\sigma(X, \mathscr{F})$-topology to some $z \in X$. Then

$$
U_{-i}\left(z_{i_{\lambda}}\right)=y_{i_{\lambda}}-z_{i_{\lambda}} \rightarrow x-z \text { in } \sigma(X, \mathscr{F}),
$$

and since the operator $U_{-i}$ is $\sigma(X, \mathscr{F})$-closed $\left(\left[2\right.\right.$, th. 2.4]), it follows that $z \in \mathscr{D}_{U_{-i}}$ and

$$
\begin{gathered}
U_{-i}(z)=x-z, \\
x=\left(1+U_{-i}\right)(z) .
\end{gathered}
$$

We conclude that $1+U_{-i}$ is surjective. By [2, lemma 3.1] it is also injective, so $-1 \notin \sigma\left(U_{-i}\right)$.

(1.5) Corollary. Let $X$ be a complex Banach space, $\mathscr{F}$ its dual, and $U$ a $\sigma(X, \mathscr{F})$ continuous, hence strongly continuous, one-parameter group of isometries in $B_{\mathscr{F}}(X)=$ $B(X)$. If there exist $P \in B(X)$ and $\lambda, \mu \in \mathbb{R}, \lambda \leq \mu$, such that $P(x)=x$ for $x \in X^{U}((-\infty, \lambda]), P(X) \subset X^{U}((-\infty, \mu])$, then we have for the spectrum $\sigma\left(U_{-i}\right)$ of the analytic generator $U_{-i}$ of $U$,

$$
\sigma\left(U_{-i}\right) \neq \mathbb{C} .
$$

Proof. Let us consider the dual group $U^{*}=U^{\mathscr{F}}$ and the adjoint $P^{*}=P^{\mathscr{F}}$ of $P$.

Let $\phi \in \mathscr{F}^{U^{*}}([\mu+1,+\infty))$. By [11, prop. 2.3.4] or [4, prop. 3.13] we have for all $x \in X$

$$
\left\langle x, P^{*}(\phi)\right\rangle=\langle P(x), \phi\rangle=0,
$$

so

$$
\left(1-P^{*}\right)(\phi)=\phi
$$

Now let $\phi \in \mathscr{F}$ be arbitrary. If $x \in X^{U}((-\infty, \lambda])$ then

$$
\left\langle x,\left(1-P^{*}\right)(\phi)\right\rangle=\langle(1-P)(x), \phi\rangle=0 .
$$

Again using [11, prop. 2.3.4] or [4, prop. 3.13], we get

$$
\left(1-P^{*}\right)(\phi) \in \mathscr{F}^{U^{*}}([\lambda,+\infty)) \text {. }
$$

Applying corollary (1.4) to the group $\mathbb{R} \ni t \mapsto U_{-t}^{*} \in B_{X}(\mathscr{F})$ and to $1-P^{*} \in B(\mathscr{F})$, we deduce that $\sigma\left(U_{i}^{*}\right) \neq \mathbb{C}$. Since $U_{i}^{*}$ is injective and $\left(U_{i}^{*}\right)^{-1}=U_{-i}^{*}([2$, th. 2.4]), it follows that $\sigma\left(U_{-i}^{*}\right) \neq \mathbb{C}$. Finally, since $U_{-i}^{*}$ is the adjoint of $U_{-i}([16$, th. 1.1]), we conclude that

$$
\sigma\left(U_{-i}\right) \neq \mathbb{C}
$$

2. The projection problem in the case of factors and prime $C^{*}$-algebras

We recall that if $(X, \mathscr{F})$ is a dual pair of Banach spaces, $U$ a $\sigma(X, \mathscr{F})$-continuous one-parameter group of isometries in $B_{\mathscr{F}}(X)$, and $D \subset \mathbb{R}$ an open set, then one defines

$$
X^{U}(D)=\sigma(X, \mathscr{F}) \text {-closure of } \underset{F \subset D \text { closed }}{\bigcup} X^{U}(F)
$$

We begin with an adaptation of $[5$, th. 2.3$]$ to the present frame:

(2.1) LEMMA. Let $(M, \alpha)$ be a one-parameter $W^{*}$-dynamical system and let us assume that there exists $P \in B(M)$ with $P(x)=x$ for $x$ in the norm-closure of 
$\bigcup_{K \subset(-\infty, 0) \text { compact }} M^{\alpha}(K), P(x) \in M^{\alpha}((-\infty, 0])$ for $x \in M$ with $\mathbb{R} \ni t \mapsto \alpha_{t}(x) \in M$ norm-continuous. Then for each compact set $K \subset(-\infty, 0)$ and each $x \in M^{\alpha}(K)$ with $\|x\|<1$ we have

$$
\|\ln (1-x)\| \leq \pi\|P\|,
$$

where $\ln : \mathbb{C} \backslash(-\infty, 0] \rightarrow \mathbb{C}$ is the analytic function defined by

$$
\ln \left(r e^{i \theta}\right)=\ln r+i \theta, \quad r>0,|\theta|<\frac{\pi}{2} .
$$

Proof. By theorem (1.3) there exists $P^{\alpha} \in B(M)$ such that:

$$
\begin{aligned}
& \left\|P^{\alpha}\right\| \leq\|P\| ; \\
& P^{\alpha} \alpha_{t}=\alpha_{t} P^{\alpha}, t \in \mathbb{R} ; \\
& P^{\alpha}(x)=x \text { for } x \text { in the norm-closure of } \bigcup_{K \subset(-\infty, 0) \text { compact }} M^{\alpha}(K), \\
& P^{\alpha}(x) \in M^{\alpha}((-\infty, 0]) \text { for } x \in M \text { with } \mathbb{R} \ni t \mapsto \alpha_{t}(x) \in M \text { norm-continuous. }
\end{aligned}
$$

Let $K \subset(-\infty, 0)$ be compact and let $x \in M^{\alpha}(K)$ with $\|x\|<1$. Using the von Neumann inequality $([13, \S 153$, th. B or Appendix, $\S 4])$ as in the proof of $[5$, th. 2.3], we deduce that $\pi / 2 \pm \operatorname{Im} \ln (1-x) \geq 0$. Therefore

$$
\|\operatorname{Im} \ln (1-x)\| \leq \frac{\pi}{2}
$$

On the other hand, by known properties of the spectral subspaces of $\alpha$ we have

$$
x^{k} \in M^{\alpha}(\underbrace{K+\cdots+K}_{k}), \quad\left(x^{*}\right)^{k} \in M^{\alpha}(\underbrace{K-\cdots-K}_{k}), \quad k \geq 1 .
$$

Since $\underbrace{K+\cdots+K}_{k} \subset(-\infty, 0)$ for all $k \geq 1$, we have

$$
P^{\alpha}(\ln (1-x))=\sum_{k=1}^{\infty} \frac{1}{k} P^{\alpha}\left(x^{k}\right)=\sum_{k=1}^{\infty} \frac{1}{k} x^{k}=\ln (1-x) .
$$

Further, since for each $k \geq 1, \mathbb{R} \ni t \mapsto \alpha_{t}\left(\left(x^{*}\right)^{k}\right) \in M$ is norm-continuous, $P^{\alpha}$ commutes with all $\alpha_{t}$, and $P^{\alpha}$ is bounded, we have

$$
P^{\alpha}\left(\left(x^{*}\right)^{k}\right) \in M^{\alpha}((-\infty, 0]) \cap M^{\alpha}(\underbrace{K-\cdots-K}_{k})=\{0\}, \quad k \geq 1 .
$$

Thus

$$
P^{\alpha}\left((\ln (1-x))^{*}\right)=\sum_{k=1}^{\infty} \frac{1}{k} P^{\alpha}\left(\left(x^{*}\right)^{k}\right)=0
$$

We conclude that

$$
\begin{aligned}
\ln (1-x) & =P^{\alpha}\left(\ln (1-x)-(\ln (1-x))^{*}\right) \\
& =2 i P^{\alpha}(\operatorname{Im} \ln (1-x)),
\end{aligned}
$$

and so

$$
\begin{aligned}
\|\ln (1-x)\| & \leq 2\left\|P^{\alpha}\right\|\|\operatorname{Im} \ln (1-x)\| \\
& \leq \pi\|P\| .
\end{aligned}
$$


Next we characterize all one-parameter $W^{*}$-dynamical systems $(M, \alpha)$, with $M$ a factor, which allow bounded linear projections onto $M^{\alpha}((-\infty, 0])$ :

(2.2) TheOREM. Let $(M, \alpha)$ be a one-parameter $W^{*}$-dynamical system with $M a$ factor. Then the following statements are equivalent:

(i) there exists $P \in B(M)$ such that $P(x)=x$ for $x$ in the norm-closure of $\bigcup_{K \subset(-\infty, 0) \text { compact }} M^{\alpha}(K), P(x) \in M^{\alpha}((-\infty, 0])$ for $x \in M$ with $\mathbb{R} \ni t \mapsto \alpha_{t}(x) \in M$ norm-continuous;

(ii) for every closed subset $F$ of $\mathbb{R}$ there exists a projection $P_{F}^{\alpha} \in B_{M_{*}}(M)$ with:

$$
\begin{aligned}
& \text { image of } P_{F}^{\alpha}=M^{\alpha}(F) ; \\
& \text { kernel of } P_{F}^{\alpha}=M^{\alpha}(\mathbb{R} \backslash F) ; \\
& P_{F}^{\alpha} \alpha_{t}=\alpha_{t} P_{F}^{\alpha}, t \in \mathbb{R} ; \text { and } \\
& \sup _{F \subset \mathbb{R} \text { closed }}\left\|P_{F}^{\alpha}\right\|<+\infty ;
\end{aligned}
$$

(iii) $\sigma(\alpha)$ is finite.

Moreover, if the above equivalent statements hold then

$$
\sup _{F \subset \text { closed }}\left\|P_{F}^{\alpha}\right\| \leq|\sigma(\alpha)| \leq e^{2} \exp (2 \pi\|P\|) .
$$

Proof. The implication (iii) $\Rightarrow$ (ii) and the inequality

$$
\sup _{F \subset \mathbb{R} \text { closed }}\left\|P_{F}^{\alpha}\right\| \leq|\sigma(\alpha)|
$$

follow immediately from corollary 1.2 . Since the implication (ii) $\Rightarrow$ (i) is trivial, it remains to show $(\mathrm{i}) \Rightarrow($ iii) and

$$
|\sigma(\alpha)| \leq e^{2} \exp (2 \pi\|P\|) .
$$

Let us therefore assume that (i) holds, and show that $|\sigma(\alpha)| \leq e^{2} \exp (2 \pi\|P\|)$. By corollary $1.4, \sigma\left(\alpha_{-i}\right) \neq \mathbb{C}$, so by [5, cor. 3.4], $\alpha$ is uniformly continuous. It follows that there exists a self-adjoint $a \in M$ with

$$
\alpha_{t}(x)=\exp (\text { ita }) x \exp (-i t a), \quad t \in \mathbb{R}, x \in M,
$$

(see e.g. $[12,8.5 .5]$ ). Since $\sigma(\alpha) \subset \sigma(a)-\sigma(a)$ (see e.g. [4, 6.19(iii)]), it suffices to show that

$$
|\sigma(a)| \leq e \exp (\pi\|P\|)
$$

Thus, let distinct points $\lambda_{1}<\lambda_{2}<\cdots<\lambda_{n}$ be in the spectrum $\sigma(a)$ of $a$. Choose some

$$
0<\varepsilon<\frac{1}{3} \min _{1 \leq j \leq n-1}\left(\lambda_{j+1}-\lambda_{j}\right)
$$

For each $1 \leq j \leq n$ let $e_{j}$ be the spectral projection of $a$ corresponding to the interval $\left[\lambda_{j}-\varepsilon, \lambda_{j}+\varepsilon\right]$. Then $0 \neq e_{j} \in M, 1 \leq j \leq n$, the projections $e_{1}, \ldots, e_{n}$ are mutually orthogonal, and by the comparability theorem (see e.g. [15, theorem 2.1.3]), there is a permutation $\tau$ of $\{1, \ldots, n\}$ with

$$
e_{\tau(1)}<e_{\tau(2)}<\cdots<e_{\tau(n)} .
$$


Consequently there are projections $f_{1}, \ldots, f_{n} \in M$ such that

$$
\begin{array}{ccc}
f_{1} \sim f_{2} \sim \cdots & f_{n} \sim e_{\tau(1)} \neq 0 . \\
\| & \| & \| M \\
e_{1} & e_{2} & e_{n}
\end{array}
$$

For any $1 \leq j \leq n-1$, let $u_{j} \in M$ be a partial isometry with

$$
u_{j}^{*} u_{j}=f_{j+1}, \quad u_{j} u_{j}^{*}=f_{j}
$$

and put

$$
u=\sum_{j=1}^{n-1} u_{j} \in M
$$

For each $1 \leq j \leq n-1$ and $k \geq 1$ we have

$$
\begin{aligned}
\left\|\alpha_{-k i}\left(u_{j}\right)\right\| & =\left\|\exp (k a) e_{j} u_{j} e_{j+1} \exp (-k a)\right\| \\
& \leq\left\|\exp (k a) e_{j}\right\|\left\|\exp (-k a) e_{j+1}\right\| \\
& \leq e^{k\left(\lambda_{j}+\varepsilon\right)} e^{-k\left(\lambda_{j+1}-\varepsilon\right)}=e^{-k\left(\lambda_{j+1}-\lambda_{j}-2 \varepsilon\right)} \leq e^{-k \varepsilon},
\end{aligned}
$$

and, similarly,

$$
\left\|\alpha_{k i}\left(u_{j}\right)\right\| \leq e^{k\left(\lambda_{j+1}-\lambda_{j}+2 \varepsilon\right)} \leq e^{k\left(\lambda_{n}-\lambda_{1}+2 \varepsilon\right)} .
$$

By [2, cor. 5.7] it follows that $u_{j} \in M^{\alpha}\left(\left[-\left(\lambda_{n}-\lambda_{1}+2 \varepsilon\right),-\varepsilon\right]\right), 1 \leq j \leq n-1$, so $u \in M^{\alpha}\left(\left[-\left(\lambda_{n}-\lambda_{1}+2 \varepsilon\right),-\varepsilon\right]\right)$. Using lemma 2.1 we obtain for every $0<\delta<1$,

$$
\|\ln (1-\delta u)\| \leq \pi\|P\| \text {. }
$$

But $u^{n}=0$, so

$$
\ln (1-\delta u)=\delta u+\frac{\delta^{2}}{2} u^{2}+\cdots+\frac{\delta^{n-1}}{n-1} u^{n-1}, \quad 0<\delta<1,
$$

and letting $\delta$ increase to 1 in the above inequality, we get

$$
\left\|u+\frac{1}{2} u^{2}+\cdots+\frac{1}{n-1} u^{n-1}\right\| \leq \pi\|P\| .
$$

We may consider $M$ as a von Neumann algebra in some complex Hilbert space $H$ ([15, theorem 1.16.7]). Choosing $\xi_{n} \in f_{n} H$ with $\left\|\xi_{n}\right\|=1$ and defining

$$
\xi_{j}=u_{j} u_{j+1} \cdots u_{n-1}\left(\xi_{n}\right), \quad 1 \leq j \leq n-1
$$

we have $\xi_{j} \in f_{j} H$ and $\left\|\xi_{j}\right\|=1,1 \leq j \leq n$, so the norm of $\xi=\frac{1}{\sqrt{n}}\left(\xi_{1}+\cdots+\xi_{n}\right)$ is equal to 1 . Hence

$$
\left\|u \xi+\frac{1}{2} u^{2} \xi+\cdots+\frac{1}{n+1} u^{n-1} \xi\right\| \leq \pi\|P\| .
$$

But by an easy computation (see the end of the proof of [5, prop. 2.6]) we get

$$
\left\|u \xi+\frac{1}{2} u^{2} \xi+\cdots+\frac{1}{n+1} u^{n-1} \xi\right\| \geq \ln \frac{n}{e},
$$

and it follows that $\ln n / e \leq \pi\|P\|$, i.e. $n \leq e \exp (\pi\|P\|)$. This proves that $|\sigma(a)| \leq$ $e \exp (\pi\|P\|)$, from which it follows as shown above that $|\sigma(\alpha)| \leq e^{2} \exp (2 \pi\|P\|)$. 
Now we consider one-parameter $C^{*}$-dynamical systems on separable prime $C^{*}$. algebras:

(2.3) Corollary. Let $(A, \alpha)$ be a one-parameter $C^{*}$-dynamical system with $A$ a separable prime $C^{*}$-algebra and let us assume that there exists $P \in B(A)$ such that

$$
\begin{aligned}
& P(x)=x \quad \text { for } x \in A^{\alpha}((-\infty, 0)), \\
& P(A) \subset A^{\alpha}((-\infty, 0]) .
\end{aligned}
$$

Then $\sigma(\alpha)$ is finite and

$$
|\sigma(\alpha)| \leq e^{2} \exp (2 \pi\|P\|) .
$$

Proof. By a theorem of Dixmier (see e.g. [12, prop. 4.3.6]) $A$ is primitive, so it has a faithful irreducible $*$-representation $\pi: A \rightarrow B(H)$.

By corollary 1.5 and by [5, prop. 2.2], $\alpha$ can be extended to a $\sigma\left(A^{* *}, A^{*}\right)$ continuous one-parameter group $\alpha^{* *}$ of $*$-automorphisms of $A^{* *}$, with $\sigma\left(\left(\alpha^{* *}\right)_{-i}\right)=$ $\sigma\left(\alpha_{-i}\right) \neq \mathbb{C}$. It is easy to verify that the second adjoint $P^{* *} \in B_{A^{*}}\left(A^{* *}\right)$ of $P$ satisfies the conditions

$$
P^{* *}(x)=x
$$

for $x \in\left(A^{* *}\right)^{\alpha^{* *}}((-\infty, 0))=\sigma\left(A^{* *}, A^{*}\right)$-closure of $\bigcup_{K \in(-\infty, 0) \text { compact }}\left(A^{* *}\right)^{\alpha^{* *}}(K)$, and

$$
P^{* *}\left(A^{* *}\right) \subset\left(A^{* *}\right)^{\alpha * *}((-\infty, 0]) .
$$

Now $\pi$ can be extended to a normal *-representation $\tilde{\pi}: A^{* *} \rightarrow B(H)$, and there is a central projection $p$ of $A^{* *}$ such that

$$
\begin{gathered}
\operatorname{ker}(\tilde{\pi})=A^{* *}(1-p), \\
\tilde{\pi} \mid A^{* *} p: A^{* *} p \rightarrow B(H) \text { is a *-isomorphism }
\end{gathered}
$$

(see the proof of $\left[15\right.$, prop. 1.16.2]). By [5, cor. 2.4] $\left(\alpha^{* *}\right),(p)=p, t \in \mathbb{R}$, so there exists a one-parameter $W^{*}$-dynamical system $(B(H), \beta)$ with

$$
\tilde{\pi} \circ\left(\alpha^{* *}\right)_{t}=\beta_{t} \circ \tilde{\pi}, \quad t \in \mathbb{R} .
$$

The linear map

$$
Q: B(H)=\tilde{\pi}\left(A^{* *} p\right) \ni \tilde{\pi}(x p) \mapsto \tilde{\pi}\left(P^{* *}(x p)\right) \in B(H)
$$

clearly satisfies the conditions

$$
\begin{aligned}
\|Q\| & \leq\left\|P^{* *}\right\|=\|P\|, \\
Q(y) & =y \quad \text { for } y \in B(H)^{\beta}((-\infty, 0)), \\
Q(B(H)) & \subset B(H)^{\beta}((-\infty, 0]),
\end{aligned}
$$

so by theorem $2.2, \sigma(\beta)$ is finite and

$$
|\sigma(\beta)| \leq e^{2} \exp (2 \pi\|Q\|) \leq e^{2} \exp (2 \pi\|P\|) .
$$

Finally, since $\pi$ is injective and $\pi \circ \alpha_{t}=\beta_{t} \circ \pi, t \in \mathbb{R}$, we have $\sigma(\alpha) \subset \sigma(\beta)$ and the above statement concerning $\sigma(\beta)$ yields the required one. 
Finally we prove the $C^{*}$-algebra counterpart to theorem 2.2 in full generality:

(2.4) THEOREM. Let $(A, \alpha)$ be a one-parameter $C^{*}$-dynamical system with $A$ a prime $C^{*}$-algebra. Then the following statements are equivalent:

(j) there exists $P \in B(A)$ such that

$$
\begin{aligned}
& P(x)=x \quad \text { for } x \in A^{\alpha}((-\infty, 0)), \\
& P(A) \subset A^{\alpha}((-\infty, 0]) ;
\end{aligned}
$$

(ji) for every closed subset $F$ of $\mathbb{R}$ there exists a projection $P_{F}^{\alpha} \in B(A)$ with:

image of $P_{F}^{\alpha}=A^{\alpha}(F)$;

kernel of $P_{F}^{\alpha}=A^{\alpha}(\mathbb{R} \backslash F)$;

$P_{F}^{\alpha} \alpha_{t}=\alpha_{t} P_{F}^{\alpha}, t \in \mathbb{R} ;$ and

$\sup _{F \subset \mathbb{R} \text { closed }}\left\|P_{F}^{\alpha}\right\|<+\infty$;

(jij) $\sigma(\alpha)$ is finite.

Moreover, if the above equivalent statements hold then

$$
\sup _{F \subset \mathbb{R} \text { closed }}\left\|P_{F}^{\alpha}\right\| \leq|\sigma(\alpha)| \leq e^{2} \exp (2 \pi\|P\|) .
$$

Proof. As in the proof of theorem 2.2, the implication $(\mathrm{jjj}) \Rightarrow(\mathrm{jj})$ and the inequality $\sup _{F \subset \mathbf{R} \text { closed }}\left\|P_{F}^{\alpha}\right\| \leq|\sigma(\alpha)|$ follow by corollary 1.2 , and the implication $(\mathrm{jj}) \Rightarrow(\mathrm{j})$ is trivial. In order to prove $(\mathrm{j}) \Rightarrow(\mathrm{jjj})$ and the inequality

$$
|\sigma(\alpha)| \leq e^{2} \exp (2 \pi\|P\|)
$$

let us assume that $(\mathrm{j})$ holds and show that $|\sigma(\alpha)| \leq e^{2} \exp (2 \pi\|P\|)$. Thus, consider $n$ distinct points in $\sigma(\alpha)$. Then by the definition of $\sigma(\alpha)$ there are $f_{1}, \ldots, f_{n} \in L^{1}(\mathbb{R})$ with

$$
1 \leq j_{1}, j_{2} \leq n, \quad j_{1} \neq j_{2} \Rightarrow \operatorname{supp}\left(\hat{f}_{j_{1}}\right) \cap \operatorname{supp}\left(\hat{f}_{j_{2}}\right)=\varnothing
$$

and $x_{1}, \ldots, x_{n} \in A$ such that

$$
\alpha_{f_{j}}\left(x_{j}\right) \neq 0 \quad \text { for all } 1 \leq j \leq n .
$$

By [5, cor. 3.2] there exists an $\alpha$-invariant separable prime $C^{*}$-subalgebra $B_{1}$ of $A$ with $B_{1} \supset\left\{x_{1}, \ldots, x_{n}\right\}$. Next, again by [5, cor. 3.2], there exists an $\alpha$-invariant separable prime $C^{*}$-subalgebra $B_{2}$ of $A$ with $B_{2} \supset B_{1} \cup P\left(B_{1}\right)$. Continuing in this way we get a sequence $B_{1} \subset B_{2} \subset \cdots$ of $\alpha$-invariant separable prime $C^{*}$-subalgebras of $A$ such that

$$
\begin{gathered}
\left\{x_{1}, \ldots, x_{n}\right\} \subset B_{1}, \\
B_{k} \cup P\left(B_{k}\right) \subset B_{k+1} \quad \text { for } k \geq 1 .
\end{gathered}
$$

Then the closure $B$ of $\bigcup_{k \geq 1} B_{k}$ is an $\alpha$-invariant separable prime $C^{*}$-subalgebra of $A$ such that $\left\{x_{1}, \ldots, x_{n}\right\} \subset B$ and $P(B) \subset B$. Applying corollary 2.3 to the restriction $\alpha \mid B$ of $\alpha$ to $B$ and to $P \mid B$, we get

$$
|\sigma(\alpha \mid B)| \leq e^{2} \exp (2 \pi\|P \mid B\|) \leq e^{2} \exp (2 \pi\|P\|) .
$$

But we have for each $1 \leq j \leq n,(\alpha \mid B)_{f_{j}}\left(x_{j}\right)=\alpha_{f_{j}}\left(x_{j}\right) \neq 0,(\alpha \mid B)_{f_{j}} \neq 0$, so

$$
\sigma(\alpha \mid B) \cap \operatorname{supp}\left(\hat{f}_{j}\right) \neq \varnothing \text {. }
$$


Thus $\sigma(\alpha \mid B)$ contains at least $n$ distinct points, and so

$$
n \leq e^{2} \exp (2 \pi\|P\|),
$$

as desired.

\section{The projection problem in the general case}

Let $M$ be a $W^{*}$-algebra, $Z$ its centre and $\Omega$ the maximal ideal space of $Z$. Following [6], we consider for each $\omega \in \Omega$ the norm-closed (automatically two-sided) ideal [ $\omega$ ] of $M$ generated by $\omega$. Denote by $x_{\omega}$ the canonical image of $x \in M$ in the quotient $C^{*}$-algebra $M /[\omega]$. We recall that by the formula from [6, p. 232],

$$
\|x\|=\sup _{\omega \in \Omega}\left\|x_{\omega}\right\|, \quad x \in M,
$$

and by [6, lemma 10], $\Omega \ni \omega \mapsto\left\|x_{\omega}\right\|$ is continuous for each $x \in M$. We note also, even if we do not use it, that each $M /[\omega]$ is a primitive $C^{*}$-algebra ([8, theorem 4.7]).

Theorem 2.2 can be extended to general one-parameter $W^{*}$-dynamical systems:

(3.1) THEOREM. Let $(M, \alpha)$ be a one-parameter $W^{*}$-dynamical system, $Z$ the centre of $M$, and $\Omega$ the maximal ideal space of $Z$. Then the following statements are equivalent:

(i) there exists $P \in B(M)$ such that $P(x)=x$ for $x$ in the norm-closure of $\bigcup_{K \in(-\infty, 0) \text { compact }} M^{\alpha}(K), P(x) \in M^{\alpha}((-\infty, 0])$ for $x \in M$ with $\mathbb{R} \ni t \mapsto \alpha_{t}(x) \in M$ norm-continuous ;

(ii) there exists $Q \in B(M)$ such that $Q(x)=x$ for $x$ in the norm-closure of $\bigcup_{K \subset(-\infty, 0) \text { compact }} M^{\alpha}(K), Q(x)=0$ for $x$ in the norm-closure of $\bigcup_{K \subset(0,+\infty) \text { compact }} M^{\alpha}(K)$;

(iii) for every closed subset $F$ of $\mathbb{R}$ there exists a projection $P_{F}^{\alpha} \in B_{M_{*}}(M)$ with: image of $\dot{P}_{F}^{\alpha}=M^{\alpha}(F)$;

kernel of $P_{F}^{\alpha}=M^{\alpha}(\mathbb{R} \backslash F)$;

$P_{F}^{\alpha} \alpha_{t}=\alpha_{t} P_{F}^{\alpha}, t \in \mathbb{R} ;$ and

$\sup _{F \subset \mathbf{R} \text { closed }}\left\|P_{F}^{\alpha}\right\|<+\infty$;

(iv) there exists a dense open subset $\mathscr{D}$ of $\Omega$ such that, for each $\omega \in \mathscr{D},[\omega]$ is $\alpha$-invariant and $\alpha$ induces a uniformly continuous one-parameter group $\alpha^{\omega}$ of *-automorphisms of the quotient $C^{*}$-algebra $M /[\omega]$, and $\sup _{\omega \in \mathscr{D}}\left|\sigma\left(\alpha^{\omega}\right)\right|<+\infty$;

(v) for each $x \in M$ and each $\phi \in M_{*}$

$$
\sup _{0<\varepsilon<\delta<+\infty}\left|\int_{\varepsilon \leq|t| \leq \delta} \frac{1}{t}\left\langle\alpha_{t}(x), \phi\right\rangle d t\right|<+\infty ;
$$

(vi) $\alpha$ has the Hilbert transform property from $[18, \S 3]$ in every $x \in M$; that is, the limit

$$
H^{\alpha}(x)=\frac{i}{\pi} \sigma\left(M, M_{*}\right)-\lim _{\substack{0<\varepsilon \rightarrow 0 \\ \delta \rightarrow+\infty}} \int_{\varepsilon \leq|t| \leq \delta} \frac{1}{t} \alpha_{t}(x) d t
$$

exists for each $x \in M$;

(vii) $\alpha$ has the global Hilbert transform property from $[18, \S 3]$; that is, it has the Hilbert transform property in every $x \in M$ and $H^{\alpha} \in B_{M_{*}}(M)$. 
Moreover, if the above equivalent statements hold then:

$$
\sup _{F \subset \mathbf{R} \text { closed }}\left\|P_{F}^{\alpha}\right\| \leq \sup _{\omega \in \mathscr{D}}\left|\sigma\left(\alpha^{\omega}\right)\right| \leq e^{2} \exp (2 \pi\|P\|) ;
$$

for given $Q, P$ can be chosen with $\|P\| \leq\|Q\| ; Q$ can be chosen with $\|Q\| \leq \frac{1}{2}\left(1+\left\|H^{\alpha}\right\|\right)$; $\left\|H^{\alpha}\right\| \leq 2\left\|P_{(-\infty, 0]}^{\alpha}\right\|$.

Proof. (i) $\Rightarrow$ (iv) and the inequality $\sup _{\omega \in \mathscr{D}}\left|\sigma\left(\alpha^{\omega}\right)\right| \leq e^{2} \exp (2 \pi\|P\|)$ :

By corollary 1.4, $\sigma\left(\alpha_{-i}\right) \neq C$, so by $\left[5\right.$, theorem 4.4], there exists a family $\left(p_{i}\right)_{i \in I}$ of mutually orthogonal central projections in $M$ with $\sum_{i \in I} p_{i}=1$ such that, for each $i \in I, p_{i}$ is fixed by $\alpha$ and $\alpha$ induces a uniformly continuous one-parameter group $\alpha \mid M_{p_{i}}$ of $*$-automorphisms of $M_{p_{i}}$. It follows that for each $i \in I$ there exists a self-adjoint $a_{i} \in M$ with

$$
\alpha_{i}(x)=\exp \left(i t a_{i}\right) x \exp \left(-i t a_{i}\right), \quad t \in \mathbb{R}, x \in M_{p_{i}}
$$

(see e.g. $[12,8.5 .5]$ ). Each $p_{i}$ corresponds to the characteristic function of some closed and open subset $K_{i}$ of $\Omega$, and the open set $\mathscr{D}=\bigcup_{i \in I} K_{i}$ is dense in $\Omega$.

Let $\omega \in \mathscr{D}$ be arbitrary. Then there exists an $i \in I$ with $\omega \in K_{i}$. Plainly, [ $\left.\omega\right]$ is $\alpha$-invariant and $\alpha$ induces a uniformly continuous one-parameter group $\alpha^{\omega}$ of *-automorphisms of $M /[\omega]$. Since

$$
\sigma\left(\alpha^{\omega}\right) \subset \sigma\left(\left(a_{i}\right)_{\omega}\right)-\sigma\left(\left(a_{i}\right)_{\omega}\right)
$$

(see e.g. $[4,6.19(\mathrm{iii})]),\left|\sigma\left(\alpha^{\omega}\right)\right| \leq e^{2} \exp (2 \pi\|P\|)$ will follow from the inequality $\left|\sigma\left(\left(a_{i}\right)_{\omega}\right)\right| \leq e \exp (\pi\|P\|)$.

In order to prove the last inequality, we proceed much as in the proof of theorem 2.2; therefore we only sketch the reasoning:

Consider $n$ distinct points $\lambda_{1}<\lambda_{2}<\cdots<\lambda_{n}$ in the spectrum of $\left(\alpha_{i}\right)_{\omega}$. Choosing some

$$
0<\varepsilon<\frac{1}{3} \min _{1 \leq j \leq n-1}\left(\lambda_{j+1}-\lambda_{j}\right),
$$

for each $1 \leq j \leq n$, let $e_{j} \in M$ be the spectral projection of $a_{i}$ corresponding to $\left[\lambda_{j}-\varepsilon, \lambda_{j}+\varepsilon\right]$. Then the projections $e_{1}, \ldots, e_{n}$ are mutually orthogonal and $\left(e_{j}\right)_{\omega} \neq 0$, $1 \leq j \leq n$. By the comparability theorem (see e.g. [15, theorem 2.1.3]) there exists, for any distinct $1 \leq j_{1}, j_{2} \leq n$, a projection $p_{j_{1}, j_{2}} \leq p_{i}$ in $Z$, corresponding to the characteristic function of some closed and open set containing $\omega$, such that either $e_{j_{1}} p_{j_{1}, j_{2}}<e_{j_{2}} p_{j_{1}, j_{2}}$, or $e_{j_{1}} p_{j_{1}, j_{2}}>e_{j_{2}} p_{j_{1}, j_{2}}$. Consequently, there is a permutation $\tau$ of $\{1, \ldots, n\}$ and a projection $p \leq p_{i}$ in $Z$, corresponding to the characteristic function of some closed and open set containing $\omega$, such that

$$
e_{\tau(1)} p<e_{\tau(2)} p<\cdots<e_{\tau(n)} p .
$$

Hence there exist projections $f_{1}, \ldots, f_{n} \in M$ such that

$$
\begin{array}{lll}
f_{1} \sim f_{2} \sim \cdots & \sim f_{n} \sim e_{\tau(1)} p \notin[\omega] . \\
\| & \| & \| \\
e_{1} & e_{2} & e_{n}
\end{array}
$$

For any $1 \leq j \leq n-1$, let $u_{j} \in M$ be a partial isometry with

$$
u_{j}^{*} u_{j}=f_{j+1}, \quad u_{j} u_{j}^{*}=f_{j},
$$


and put

$$
u=\sum_{j-1}^{n-1} u_{j} \in M
$$

Using [2, cor. 5.7], it is easy to see that $u_{j} \in M^{\alpha}\left(\left[-\left(\lambda_{n}-\lambda_{1}+2 \varepsilon\right),-\varepsilon\right]\right), 1 \leq j \leq n-1$, so $u \in M^{\alpha}\left(\left[-\left(\lambda_{n}-\lambda_{1}+2 \varepsilon\right),-\varepsilon\right]\right)$. By lemma 2.1 it follows that

$$
\begin{aligned}
\left\|u_{\omega}+\frac{1}{2}\left(u_{\omega}\right)^{2}+\cdots+\frac{1}{n-1}\left(u_{\omega}\right)^{n-1}\right\| & \leq\left\|u+\frac{1}{2} u^{2}+\cdots+\frac{1}{n-1} u^{n-1}\right\| \\
& =\lim _{(0,1) \ni \delta \rightarrow 1}\|\ln (1-\delta u)\| \leq \pi\|P\| .
\end{aligned}
$$

But on the other hand, each $\left(f_{j}\right)_{\omega}$ being non-zero, considering $M /[\omega]$ as a $C^{*}$. subalgebra of $B(H)$ for some complex Hilbert space $H$ (see e.g. [15, theorem 1.16.6]) and computing as at the end of the proof of theorem 2.2, we get

$$
\left\|u_{\omega}+\frac{1}{2}\left(u_{\omega}\right)^{2}+\cdots+\frac{1}{n-1}\left(u_{\omega}\right)^{n-1}\right\| \geq \ln \frac{n}{e}
$$

Thus $\ln n / e \leq \pi\|P\|, n \leq e \exp (\pi\|P\|)$, as desired.

(iv) $\Rightarrow$ (iii) and the inequality $\sup _{F \subset \mathbf{R} \text { closed }}\left\|P_{F}^{\alpha}\right\| \leq \sup _{\omega \in \mathscr{D}}\left|\sigma\left(\alpha^{\omega}\right)\right|$ :

Set $n_{0}=\sup _{\omega \in \mathscr{D}}\left|\sigma\left(\alpha^{\omega}\right)\right|$, and let $F$ be an arbitrary closed subset of $\mathbb{R}$. By [5, theorem 4.4 and cor. 2.4], $\alpha_{t}(z)=z$ for all $z \in Z$ and $t \in \mathbb{R}$. Using Zorn's lemma, we get a maximal family of mutually orthogonal non-zero projections $\left(p_{i}\right)_{i \in I}$ in $Z$ such that for each $i \in I$ there exists a projection $P_{F}^{\alpha \mid M p_{i}} \in B_{\left(M p_{i}\right)_{*}}\left(M p_{i}\right)$ with:

image of $P_{F}^{\alpha \mid M P_{i}}=\left(M p_{i}\right)^{\alpha \mid M p_{i}}(F)=M^{\alpha}(F) p_{i}$;

kernel of $P_{F}^{\alpha \mid M p_{i}}=\left(M p_{i}\right)^{\alpha \mid M p_{i}}(\mathbb{R} \backslash F)=M^{\alpha}(\mathbb{R} \backslash F) p_{i}$;

$P_{F}^{\alpha \mid M p_{i}}\left(\alpha \mid M p_{i}\right)_{t}=\left(\alpha \mid M p_{i}\right)_{t} P_{F}^{\alpha \mid M p_{i}}, \quad t \in \mathbb{R} ;$

$\left\|P_{F}^{\alpha \mid M p_{i}}\right\| \leq n_{0}$.

If we show that $\sum_{i \in I} p_{i}=1$, then $P_{F}^{\alpha}=\sum_{i \in I} P_{F}^{\alpha \mid M p_{i}}$ would be a projection in $B_{M_{*}}(M)$ with:

image of $P_{F}^{\alpha}=M^{\alpha}(F)$;

kernel of $P_{F}^{\alpha}=M^{\alpha}(\mathbb{R} \backslash F)$;

$P_{F}^{\alpha} \alpha_{t}=\alpha_{t} P_{F}^{\alpha}, \quad t \in \mathbb{R}$;

$\left\|P_{F}^{\alpha}\right\| \leq n_{0}$.

Therefore let $p_{0}=1-\sum_{i \in I} p_{i}$ and let us prove that the assumption $p_{0} \neq 0$ leads to a contradiction.

Let us identify each projection $p \in Z$ with the characteristic function of the closed and open subset $\left\{\omega \in \Omega ; p_{\omega}=1_{\omega}\right\}$ of $\Omega$. So $p_{0}$ is the characteristic function $\chi_{K_{0}}$ of some closed and open set $\varnothing \neq K_{0} \subset \Omega$. By [5, theorem 4.4] there is a closed and open set $\varnothing \neq K_{1} \subset K_{0} \cap \mathscr{D}$ such that $\alpha \mid M_{\chi_{K_{1}}}$ is uniformly continuous. Set

$$
n_{1}=\sup _{\omega \in K_{1}}\left|\sigma\left(\alpha^{\omega}\right)\right| \leq n_{0}
$$

Choosing some $\omega_{1} \in K_{1}$ with $\left|\sigma\left(\alpha^{\omega_{1}}\right)\right|=n_{1}$, we claim that there is an open neighbourhood $D \subset K_{1}$ of $\omega_{1}$ with

$$
\omega \in D \Rightarrow\left|\sigma\left(\alpha^{\omega}\right)\right|=n_{1}
$$


Indeed, there are $f_{1}, \ldots, f_{n_{1}} \in L^{1}(\mathbb{R})$ with $\operatorname{supp}\left(\hat{f}_{1}\right), \ldots, \operatorname{supp}\left(\hat{f}_{n_{1}}\right)$ mutually disjoint and $x_{1}, \ldots, x_{n_{1}} \in M \chi_{K_{1}}$ such that

$$
\left(\alpha_{f_{1}}\left(x_{1}\right)\right)_{\omega_{1}}, \ldots,\left(\alpha_{f_{n_{1}}}\left(x_{n_{1}}\right)\right)_{\omega_{1}} \neq 0,
$$

and by [6, lemma 10] we have for all $\omega$ in some open neighbourhood $D \subset K_{1}$ of $\omega_{1}$

$$
\left(\alpha_{f_{1}}\left(x_{1}\right)\right)_{\omega}, \ldots,\left(\alpha_{f_{n_{1}}}\left(x_{n_{1}}\right)\right)_{\omega} \neq 0 \text {. }
$$

It follows that there exists a closed and open set $\varnothing \neq K_{2} \subset K_{1}$ such that $\omega \in K_{2} \Rightarrow$ $\left|\sigma\left(\alpha^{\omega}\right)\right|=n_{1}$. For each $\omega \in K_{2}$, set

$$
\sigma\left(\alpha^{\omega}\right)=\left\{\lambda_{1}(\omega), \ldots, \lambda_{n_{1}}(\omega)\right\}
$$

where $\lambda_{1}(\omega)<\cdots<\lambda_{n_{1}}(\omega)$. We claim that for each $1 \leq j \leq n_{1}$ the function $K_{2} \ni \omega \mapsto$ $\lambda_{j}(\omega)$ is continuous. To prove this, let $\omega_{2} \in K_{2}$ and let

$$
0<\varepsilon<\frac{1}{2} \min _{1 \leq j \leq n_{1}-1}\left(\lambda_{j+1}\left(\omega_{2}\right)-\lambda_{j}\left(\omega_{2}\right)\right)
$$

be arbitrary. Then there are $f_{1}, \ldots, f_{n_{1}} \in L^{1}(\mathbb{R})$ with

$$
\operatorname{supp}\left(\hat{f}_{j}\right) \subset\left(\lambda_{j}\left(\omega_{2}\right)-\varepsilon, \lambda_{j}\left(\omega_{2}\right)+\varepsilon\right)
$$

and $x_{1}, \ldots, x_{n_{1}} \in M_{\chi_{K_{2}}}$ such that

$$
\left(\alpha_{f_{1}}\left(x_{1}\right)\right)_{\omega_{2}}, \ldots,\left(\alpha_{f_{n_{1}}}\left(x_{n_{1}}\right)\right)_{\omega_{2}} \neq 0 .
$$

Again by [6, lemma 10], there exists an open neighbourhood $D \subset K_{2}$ of $\omega_{2}$ such that for every $\omega \in D$

$$
\left(\alpha_{f_{1}}\left(x_{1}\right)\right)_{\omega}, \ldots,\left(\alpha_{f_{n_{1}}}\left(x_{n_{1}}\right)\right)_{\omega} \neq 0 .
$$

For each $\omega \in D$ we get, successively,

$$
\begin{aligned}
\sigma\left(\alpha^{\omega}\right) \cap \operatorname{supp}\left(\hat{f}_{j}\right) \neq \varnothing, & & 1 \leq j \leq n_{1}, \\
\sigma\left(\alpha^{\omega}\right) \cap\left(\lambda_{j}\left(\omega_{2}\right)-\varepsilon, \lambda_{j}\left(\omega_{2}\right)+\varepsilon\right) \neq \varnothing, & & 1 \leq j \leq n_{1}, \\
\left|\lambda_{j}(\omega)-\lambda_{j}\left(\omega_{2}\right)\right|<\varepsilon, & & 1 \leq j \leq n_{1} .
\end{aligned}
$$

For each $J \subset\left\{1, \ldots, n_{1}\right\}$ and $k \geq 1$ we set

$$
K_{J, k}=\left\{\omega \in K_{2} ; \begin{array}{ll}
\lambda_{j}(\omega) \in F & \text { for } j \in J \\
d\left(\lambda_{j}(\omega), F\right)=\inf _{\lambda \in F}\left|\lambda_{j}(\omega)-\lambda\right| \geq \frac{1}{k} & \text { for } j \notin J
\end{array}\right\} .
$$

Then the $K_{J, k}$ 's are closed subsets of $K_{2}$ and $\bigcup_{J, k} K_{J, k}=K_{2}$. Using the Baire category property of $K_{2}$, we deduce that there exist $J_{0} \subset\left\{1, \ldots, n_{1}\right\}$ and $k_{0} \geq 1$ such that the interior of $K_{J_{0}, k_{0}} \neq \varnothing$. Therefore there is a closed and open subset $K_{3} \neq \varnothing$ of $\Omega$ which is contained in $K_{J_{0}, k_{0}}$. If $f \in L^{1}(\mathbb{R})$ and

$$
\operatorname{supp}(\hat{f}) \cap\left(F \cup\left\{\lambda \in \mathbb{R} ; d(\lambda, F) \geq \frac{1}{k_{0}}\right\}\right)=\varnothing,
$$

then we have

$$
\left(\alpha_{f}(x)\right)_{\omega}=\left(\alpha^{\omega}\right)_{f}\left(x_{\omega}\right)=0, \quad x \in M \chi_{K_{3}}, \omega \in K_{3},
$$

so $\left(\alpha \mid M_{\chi_{K_{3}}}\right)_{f}=0$, and consequently,

$$
\sigma\left(\alpha \mid M_{\chi_{K_{3}}}\right) \subset\left(F \cup\left\{\lambda \in \mathbb{R} ; d(\lambda, F) \geq \frac{1}{k_{0}}\right\}\right) .
$$


Set $q=\chi_{K_{3}} \in Z$. Then $q$ is a non-zero projection and is orthogonal to all $\boldsymbol{P}_{i}$. Choosing some $f_{0} \in L^{\prime}(\mathbb{R})$ such that

$\hat{f}_{0}(s)=1$ for $s$ in a neighbourhood of the compact set $F \cap \sigma(\alpha \mid M q)$,

$\operatorname{supp}\left(\hat{f}_{0}\right) \subset\left\{\lambda \in \mathbb{R} ; d(\lambda, F)<1 / k_{0}\right\}$,

and putting $P_{F}^{\alpha \mid M q}=(\alpha \mid M q)_{f_{0}} \in B_{(M q)_{*}}(M q)$, it is easy to see that $P_{F}^{\alpha \mid M q}$ is a projection with:

$$
\begin{aligned}
& \text { image of } P_{F}^{\alpha \mid M q}=(M q)^{\alpha \mid M q}(F) ; \\
& \text { kernel of } P_{F}^{\alpha \mid M q}=(M q)^{\alpha \mid M q}(\mathbb{R} \backslash F)=(M q)^{\alpha \mid M q}\left(\left\{\lambda \in \mathbb{R} ; d(\lambda, F) \geq 1 / k_{0}\right\}\right), \\
& P_{F}^{\alpha \mid M q}(\alpha \mid M q)_{t}=(\alpha \mid M q)_{t} P_{F}^{\alpha \mid M q}, \quad t \in \mathbb{R} .
\end{aligned}
$$

By corollary 1.2 we have for all $x \in M q$

$$
\left\|\left(P_{F}^{\alpha \mid M q}(x)\right)_{\omega}\right\| \leq n_{1}\left\|x_{\omega}\right\| \leq n_{0}\|x\|, \quad \omega \in K_{3},
$$

so

$$
\left\|P_{F}^{\alpha \mid M q}(x)\right\|=\sup _{\omega \in K_{3}}\left\|\left(P_{F}^{\alpha \mid M q}(x)\right)_{\omega}\right\| \leq n_{0}\|x\| \text {. }
$$

It follows that $\left\|P_{F}^{\alpha \mid M q}\right\| \leq n_{0}$. But the existence of $q$ with the above properties contradicts the maximality of the family $\left(p_{i}\right)_{i \in I}$.

(iii) $\Rightarrow$ (vii) and the inequality $\left\|H^{\alpha}\right\| \leq 2\left\|P_{(-\infty, 0]}^{\alpha}\right\|:$

By [18, theorem 3.5] the existence of $P_{(-\infty, 0]}^{\alpha}$ and $P_{[0,+\infty)}^{\alpha}$ implies the global Hilbert transform property for $\alpha$ and $H^{\alpha}=P_{(-\infty, 0]}^{\alpha}-P_{[0,+\infty)}^{\alpha}$. Since $P_{[0,+\infty)}^{\alpha}(x)=$ $\left(P_{(-\infty, 0]}^{\alpha}\left(x^{*}\right)\right)^{*}, x \in M$, we have

$$
\left\|H^{\alpha}\right\| \leq\left\|P_{(-\infty, 0]}^{\alpha}\right\|+\left\|P_{[0,+\infty)}^{\alpha}\right\|=2\left\|P_{(-\infty, 0]}^{\alpha}\right\| .
$$

The implications (vii) $\Rightarrow(\mathrm{vi}) \Rightarrow(\mathrm{v})$ are trivial.

(vii) $\Rightarrow$ (ii) and $Q$ can be chosen such that $\|Q\| \leq \frac{1}{2}\left(1+\left\|H^{\alpha}\right\|\right)$ :

By [18, lemma 3.4], $Q=\frac{1}{2}\left(1+H^{\alpha}\right) \in B_{M_{*}}(M)$ satisfies the conditions from (ii). (v) $\Rightarrow$ (ii):

For every $0<\varepsilon<\delta<+\infty$ we set

$$
T_{\varepsilon, \delta}=\int_{\varepsilon \leq|t| \leq \delta} \frac{1}{t} \alpha_{t} d t \in B_{M_{*}}(M) .
$$

By the uniform boundedness principle, $c=\sup _{0<\varepsilon<\delta<+\infty}\left\|T_{\varepsilon, \delta}\right\|<+\infty$. Now let $\mathscr{X}$ be the complex linear space of all continuous functions $f: \mathbb{R} \backslash\{0\} \rightarrow \mathbb{C}$ with

$$
p(f)=\sup _{0<\varepsilon<\delta<+\infty}\left|\int_{\varepsilon \leq|t| \leq \delta} f(t) d t\right|<+\infty .
$$

Then $p$ is a semi-norm on $\mathscr{X}$,

$$
\mathscr{Y}=\left\{f \in \mathscr{X} ; \lim _{\substack{0<\varepsilon \rightarrow 0 \\ \delta \rightarrow+\infty}} \int_{\varepsilon \leq|t| \leq \delta} f(t) d t \text { exists }\right\}
$$

a linear subspace of $\mathscr{X}$, and

$$
\mathscr{Y} \ni f \mapsto \lim _{\substack{0<\varepsilon \rightarrow 0 \\ \delta \rightarrow+\infty}} \int_{\varepsilon \leq|t| \leq \delta} f(t) d t
$$


a linear functional on $\mathscr{Y}$ with

$$
\left|\lim _{\substack{0<\varepsilon \rightarrow 0 \\ \delta \rightarrow+\infty}} \int_{\varepsilon \leq|t| \leq \delta} f(t) d t\right| \leq p(f), \quad f \in \mathscr{Y} .
$$

By the Hahn-Banach theorem there exists a linear functional

$$
\mathscr{X} \ni f \mapsto \operatorname{LiM}_{\substack{0<\varepsilon \rightarrow 0 \\ \delta \rightarrow+\infty}} \int_{\varepsilon \leq|t| \leq \delta} f(t) d t,
$$

which extends the above linear functional on $y$ and for which

$$
\left|\operatorname{LIM}_{\substack{0<\varepsilon \rightarrow 0 \\ \delta \rightarrow+\infty}} \int_{\varepsilon \leq|t| \leq \delta} f(t) d t\right| \leq p(f), \quad f \in \mathscr{X} .
$$

For any $x \in M$ and $\phi \in M_{*}$, the function

$$
f_{x, \phi}: \mathbb{R} \backslash\{0\} \ni t \mapsto \frac{1}{t}\left\langle\alpha_{t}(x), \phi\right\rangle
$$

belongs to $\mathscr{X}$ and $p\left(f_{x, \phi}\right) \leq c\|x\|\|\phi\|$. Thus

$$
M \times M_{*} \ni(x, \phi) \mapsto \operatorname{LIM}_{\substack{0<\varepsilon \rightarrow 0 \\ \delta \rightarrow+\infty}} \int_{\varepsilon \leq|t| \leq \delta} f_{x, \phi}(t) d t
$$

is a bilinear functional of norm $\leq c$. Since $M$ is the dual space of $M_{*}$, it follows that there exists $T \in B(M)$ such that

$$
\langle T(x), \phi\rangle=\operatorname{LIM}_{\substack{0<\varepsilon \rightarrow 0 \\ \delta \rightarrow+\infty}} \int_{\varepsilon \leq|t| \leq \delta} f_{x, \phi}(t) d t, \quad x \in M, \phi \in M_{*} .
$$

In particular,

$$
T(x)=\sigma\left(M, M_{*}\right)-\lim _{\substack{0<\varepsilon \rightarrow 0 \\ \delta \rightarrow+\infty}} \int_{\varepsilon \leq|t| \leq \delta} \frac{1}{t} \alpha_{t}(x) d t
$$

whenever the limit at the right side exists. But if $x \in M^{\alpha}((-\infty,-a])$ for some $a>0$, then $\lim _{\delta \rightarrow+\infty}\left\|\alpha_{-\delta i}(x)\right\|=0$, so by [18, lemma 3.1],

$$
\sigma\left(M, M_{*}\right)-\lim _{\substack{0<\varepsilon \rightarrow 0 \\ \delta \rightarrow+\infty}} \int_{\varepsilon \leq|t| \leq \delta} \frac{1}{t} \alpha_{t}(x) d t=\frac{\pi}{i} x .
$$

Thus $T(x)=(\pi / i) x$ for $x$ in the norm-closure of $\bigcup_{K \subset(-\infty, 0) \text { compact }} M^{\alpha}(K)$. One deduces similarly that $T(x)=-(\pi / i) x$ for $x$ in the norm-closure of $\bigcup_{K \subset(0,+\infty) \text { compact }} M^{\alpha}(K)$. We conclude that $Q=\frac{1}{2}(1+(i T / \pi)) \in B(M)$ satisfies the conditions from (ii).

Finally we prove that

(ii) $\Rightarrow$ (i) and, for given $Q, P$ can be chosen with $\|P\| \leq\|Q\|$ :

By theorem 1.3 there exists $Q^{\alpha} \in B(M)$ such that $\left\|Q^{\alpha}\right\| \leq\|Q\|, Q^{\alpha} \alpha_{t}=\alpha_{t} Q^{\alpha}, t \in \mathbb{R}$, $Q^{\alpha}(x)=x$ for $x$ in the norm-closure of $\bigcup_{K \subset(-\infty, 0) \text { compact }} M^{\alpha}(K)$, and $Q^{\alpha}(x)=0$ for $x$ in the norm-closure of $\bigcup_{K \subset(0,+\infty) \text { compact }} M^{\alpha}(K)$. 
Let us assume that there exists $x \in M$ with $\mathbb{R} \exists t \mapsto \alpha_{t}(x)$ norm-continuous and $Q^{\alpha}(x) \notin M^{\alpha}((-\infty, 0])$. Then there is some $f \in L^{1}(\mathbb{R})$ such that supp $(\hat{f}) \subset(0,+\infty)$ is compact, $\alpha_{f}\left(Q^{\alpha}(x)\right) \neq 0$. But this is not possible, because as $\alpha_{f}(x) \in M^{\alpha}(\operatorname{supp}(\hat{f}))$ one has $\alpha_{f}\left(Q^{\alpha}(x)\right)=Q^{\alpha}\left(\alpha_{f}(x)\right)=0$. Thus $Q^{\alpha}(x) \in M^{\alpha}((-\infty, 0])$ for $x \in M$ with $\mathbb{R} \ni t \mapsto \alpha_{t}(x)$ norm-continuous, so (i) holds with $P=Q^{\alpha}$.

(3.2) Corollary. Let $(M, \alpha)$ be a one-parameter. $W^{*}$-dynamical system, $N$ an $\alpha$-invariant $W^{*}$-subalgebra of $M$, and $\alpha \mid N$ the restriction of $\alpha$ to $N$. If $(M, \alpha)$ satisfies the equivalent conditions from theorem 3.1, then $(N, \alpha \mid N)$ also satisfies them and

$$
P_{F}^{\alpha \mid N}=P_{F}^{\alpha} \mid N, \quad F \subset \mathbb{R} \text { closed. }
$$

Proof. If $(M, \alpha)$ satisfies one of the conditions (v), (vi), (vii) from theorem 3.1, then it is plainly satisfied also by $(N, \alpha \mid N)$. Further, if $F \subset \mathbb{R}$ is closed and $x \in N$, then $P_{F}^{\alpha \mid N}(x) \in N^{\alpha \mid N}(F) \subset M^{\alpha}(F), x-P_{F}^{\alpha \mid N}(x) \in N^{\alpha \mid N}(\mathbb{R} \backslash F) \subset M^{\alpha}(\mathbb{R} \backslash F)$, so $P_{F}^{\alpha \mid N}(x)=P_{F}^{\alpha}(x)$.

For one-parameter $C^{*}$-dynamical systems we have two non-equivalent natural counterparts to theorem 3.1 ; one of them corresponds to condition (v) from theorem 3.1, and the second one to the conditions (vi) and (vii).

Let us recall that if $A$ is a $C^{*}$-algebra and Prim $(A)$ is the set of all primitive closed two-sided ideals of $A$, then Prim $(A)$ is usually endowed with the Jacobson topology (see e.g. $[12,4.1 .4]$ ). By a theorem of Dixmier Prim $(A)$ is a Baire space (see e.g. [12, theorem 4.3.5).

(3.3) LemMA. Let $(A, \alpha)$ be a one-parameter $C^{*}$-dynamical system such that any closed two-sided ideal of $A$ is $\alpha$-invariant. For each closed two-sided ideal $\mathscr{I}$ of $A$ we denote by $\alpha^{\$}$ the strongly continuous one-parameter group of *-automorphisms of the quotient $C^{*}$-algebra $A / \mathscr{I}$. Then the set

$$
\left\{\mathscr{J} \in \operatorname{Prim}(A) ; \sigma\left(\alpha^{\mathscr{I}}\right) \subset F\right\}
$$

is closed in Prim $(A)$ for any closed $F \subset \mathbb{R}$.

Proof. Let $F \subset \mathbb{R}$ be closed and let us assume that there is some $\mathscr{J}_{0}$ in the closure of $\left\{\mathscr{J} \in \operatorname{Prim}(A) ; \sigma\left(\alpha^{\mathscr{I}}\right) \subset F\right\}$ in Prim $(A)$ such that $\sigma\left(\alpha^{\mathscr{J}_{0}}\right) \not \subset F$. Choosing some $\lambda_{0} \in \sigma\left(\alpha^{J_{0}}\right) \backslash F$, and an $f \in L^{1}(\mathbb{R})$ with $\hat{f}\left(\lambda_{0}\right) \neq 0$, supp $(\hat{f}) \cap F=\varnothing$, there exists $x \in A$ such that $\alpha_{f}(x) \notin \mathscr{J}_{0}$. On the other hand, $\left(\alpha^{\mathscr{g}}\right)_{f}=0$ for every $\mathscr{g} \in \operatorname{Prim}(A)$ with $\sigma\left(\alpha^{\mathscr{J}}\right) \subset F$, so

$$
\alpha_{f}(x) \in \bigcap_{\substack{g \in \operatorname{Prim}_{(1)}(A) \\ \sigma\left(\alpha^{\xi}\right) \subset F}} \mathscr{J} \subset \mathscr{J}_{0} .
$$

This contradiction shows that the statement of the lemma holds.

If $\mathscr{I}$ is a closed two-sided ideal of a $C^{*}$-algebra $A$, then $\mathscr{J} \mapsto \mathscr{J} \cap \mathscr{I}$ is a homeomorphism of the open subset $\{\mathscr{F} \in \operatorname{Prim}(A) ; \mathscr{I} \not \subset \mathscr{J}\}$ of $\operatorname{Prim}(A)$ onto Prim $(\mathscr{I})$ (see e.g. $[12$, theorem 4.1.11]). Therefore we shall identify Prim $(\mathscr{I})$ with the above open subset of Prim $(A)$.

We also recall that a composition series for a $C^{*}$-algebra $A$ is a strictly increasing family of closed two-sided ideals $\mathscr{I}_{a}$ of $A$, indexed by a segment $\{a ; 0 \leq a \leq c\}$ of 
the ordinals, such that

$$
\begin{aligned}
& \mathscr{I}_{0}=\{0\}, \\
& \mathscr{I}_{c}=A, \\
& \mathscr{I}_{a}=\bigcup_{\ell<a} \mathscr{I}_{\ell} \quad \text { for each limit ordinal } a \leq c .
\end{aligned}
$$

We call a composition series $\left\{\mathscr{I}_{a} ; 0 \leq a \leq c\right\}$ saturated if, for each $0 \leq a<c$, $\operatorname{Prim}\left(\mathscr{I}_{a+1} / \mathscr{I}_{a}\right)$ is dense in Prim $\left(A / \mathscr{I}_{a}\right)$.

If $(A, \alpha)$ is a one-parameter $C^{*}$-dynamical system and $\mathscr{I}$ an $\alpha$-invariant closed two-sided ideal of $A$, then we denote by $\alpha \mid \mathscr{I}$ and $\alpha^{\mathscr{I}}$ the strongly continuous one-parameter groups of $*$-automorphisms of $\mathscr{I}$, resp. $A / \mathscr{I}$, induced by $\alpha$.

Now we prove a first $C^{*}$-algebra counterpart to theorem 3.1:

(3.4) TheOREM. Let $(A, \alpha)$ be a one-parameter $C^{*}$-dynamical system. Then the following statements are equivalent:

(i) there exists an $\alpha$-invariant closed two-sided ideal $\mathscr{I}$ of $A$ with Prim $(\mathscr{I})$ dense in Prim $(A)$, and $P \in B(\mathscr{Y})$ such that

$$
\begin{aligned}
& P(x)=x \quad \text { for } x \in \mathscr{I}^{\left.\alpha\right|^{\mathscr{I}}}((-\infty, 0)), \\
& P(\mathscr{I}) \subset \mathscr{I}^{\left.\alpha\right|^{\mathscr{I}}}((-\infty, 0]) ;
\end{aligned}
$$

(ii) there exists a saturated composition series $\left\{\mathscr{I}_{a} ; 0 \leq a \leq c\right\}$ for $A$, formed by, $\alpha$-invariant ideals, and projections $P_{a} \in B\left(\mathscr{I}_{a+1} / \mathscr{I}_{a}\right), 0 \leq a<c$, with

image of $P_{a}=\left(\mathscr{I}_{a+1} / \mathscr{I}_{a}\right)^{\alpha_{a}^{\psi_{a} \mid \mathscr{S}_{a+1}}{ }^{/ \mathscr{S}} a}((-\infty, 0])$,

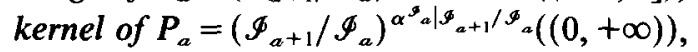

$P_{a}\left(\alpha^{\mathscr{I}} a \mid \mathscr{I}_{a+1} / \mathscr{I}_{a}\right)_{t}=\left(\alpha^{\mathscr{I}_{a}} \mid \mathscr{I}_{a+1} / \mathscr{I}_{a}\right)_{t} P_{a}, \quad t \in \mathbb{R}$,

such that

$$
\sup _{0 \leq a<c}\left\|P_{a}\right\|<+\infty
$$

(iii) there exists a family $\left(\mathscr{I}_{i}\right)_{i \in I}$ of $\alpha$-invariant norm-closed two-sided ideals of $A$ with

$$
\bigcap_{i \in I} \mathscr{I}_{i}=\{0\}, \quad \sup _{i \in I}\left|\sigma\left(\alpha^{\Phi_{i}}\right)\right|<+\infty
$$

(iv) every prime closed two-sided ideal of $A$ is $\alpha$-invariant and

$$
\sup _{\Phi_{\text {prime }}}\left|\sigma\left(\alpha^{\Phi}\right)\right|<+\infty
$$

(v) the formula

$$
\left(\alpha^{* *}\right)_{t}(x)=\left(\alpha_{t}\right)^{* *}(x), \quad t \in \mathbb{R}, x \in A^{* *}
$$

defines a $\sigma\left(A^{* *}, A^{*}\right)$-continuous one-parameter group $\alpha^{* *}$ of *automorphisms of the $W^{*}$-algebra $A^{* *}$, and $\left(A^{* *}, \alpha^{* *}\right)$ satisfies the equivalent conditions of theorem 3.1 ;

(vi) for each $x \in A$ and $\phi \in A^{*}$,

$$
\sup _{0<\varepsilon<\delta<+\infty}\left|\int_{\varepsilon \leq|r| \leq \delta} \frac{1}{t}\left\langle\alpha_{t}(x), \phi\right\rangle d t\right|<+\infty .
$$


Moreover, if the above equivalent statements hold, then

$$
\begin{aligned}
\left\|P_{(-\infty, 0]}^{\alpha^{* *}}\right\| & =\sup _{0 \leq a<c}\left\|P_{a}\right\| \leq \sup _{\mathscr{I} \in \operatorname{Prim}(A)}\left|\sigma\left(\alpha^{\mathscr{J}}\right)\right| \\
& =\sup _{\mathscr{\Phi} \text { prime }}\left|\sigma\left(\alpha^{\mathscr{I}}\right)\right| \leq \sup _{i \in I}\left|\sigma\left(\alpha^{\Phi}\right)\right|,
\end{aligned}
$$

and for suitable choice of the family $\left(\mathscr{I}_{i}\right)_{i \in I}$,

$$
\sup _{i \in I}\left|\sigma\left(\alpha^{\mathscr{s}}\right)\right| \leq e^{2} \exp (2 \pi\|P\|) \text {. }
$$

Proof. First we prove that (i) $\Rightarrow$ (iii) and, for given $P$, the $\mathscr{I}_{i}$ 's can be chosen with $\sup _{i \in I}\left|\sigma\left(\alpha^{\mathscr{J}}\right)\right| \leq e^{2} \exp (2 \pi\|P\|)$ :

Since $\bigcap_{\substack{\mathscr{g} \in \operatorname{Prim}(A) \\ \mathscr{q} \neq \mathscr{J}}} \mathscr{J}=\{0\}$, it is enough to prove that each $\mathscr{J} \in \operatorname{Prim}(A)$ with $\mathscr{I} \not \subset \mathscr{J}$ is $\alpha$-invariant and $\left|\sigma\left(\alpha^{\mathscr{I}}\right)\right| \leq e^{2} \exp (2 \pi\|P\|)$.

Let $\pi: A \rightarrow B(H)$ be an irreducible $*$-representation with $\operatorname{ker}(\pi)=\mathscr{I}$. Since $\pi(\mathscr{I})$ is a non-zero two-sided ideal of $\pi(A)$, it is dense in $B(H)$ in the strong operator topology. Therefore $\pi \mid \mathscr{I}: \mathscr{I} \rightarrow B(H)$ is an irreducible $*$-representation of $\mathscr{I}$.

Taking into account corollary 1.5 and [5, prop. 2.2 and cor. 2.4 ], by a second dual argument similar to that used in the proof of corollary 2.3 , we get a one-parameter $W^{*}$-dynamical system $(B(H), \beta)$ with

$$
(\pi \mid \mathscr{I}) \circ(\alpha \mid \mathscr{I})_{t}=\beta_{t} \circ(\pi \mid \mathscr{I}), \quad t \in \mathbb{R},
$$

and a linear map $Q: B(H) \rightarrow B(H)$ such that $\|Q\| \leq\|P\|, Q(y)=y$ for $y \in B(H)^{\beta}((-\infty, 0))$, and $Q(B(H)) \subset B(H)^{\beta}((-\infty, 0])$.

Let $a \in A$ be arbitrary. We have, for any $t \in \mathbb{R}$ and $b \in \mathscr{I}$,

$$
\begin{aligned}
\pi\left(\alpha_{t}(a)\right) \pi\left(\alpha_{t}(b)\right) & =\pi\left(\alpha_{t}(a b)\right)=\beta_{t}(\pi(a b))=\beta_{t}(\pi(a)) \beta_{t}(\pi(b)) \\
& =\beta_{t}(\pi(a)) \pi\left(\alpha_{t}(b)\right) .
\end{aligned}
$$

Since $\alpha_{t}(\mathscr{I})=\mathscr{I}$ for all $t \in \mathbb{R}$, it follows that

$$
\pi\left(\alpha_{t}(a)\right) \pi(b)=\beta_{t}(\pi(a)) \pi(b), \quad t \in \mathbb{R}, b \in \mathscr{I} .
$$

But the identity operator on $H$ belongs to the closure of $\pi(\mathscr{I})$ in the strong operator topology, so $\pi\left(\alpha_{t}(a)\right)=\beta_{t}(\pi(a)), t \in \mathbb{R}$. Consequently, $\pi \circ \alpha_{t}=\beta_{t} \circ \pi, t \in \mathbb{R}$. In particular, $\Phi=\operatorname{ker}(\pi)$ is $\alpha$-invariant. Finally, by theorem 2.2 ,

$$
|\sigma(\beta)| \leq e^{2} \exp (2 \pi\|Q\|) \leq e^{2} \exp (2 \pi\|P\|) .
$$

Since $\sigma\left(\alpha^{\mathscr{J}}\right) \subset \sigma(\beta)$, we conclude that

$$
\left|\sigma\left(\alpha^{\mathscr{J}}\right)\right| \leq e^{2} \exp (2 \pi\|P\|) .
$$

(iii) $\Rightarrow$ (iv) and the inequality $\sup _{\mathscr{I} \text { prime }}\left|\sigma\left(\alpha^{\mathscr{I}}\right)\right| \leq \sup _{i \in I}\left|\sigma\left(\alpha^{\mathscr{S}_{i}}\right)\right|$ :

We shall first show that if $J_{0}$ is a dense subset of the space $J$ of all prime closed two-sided ideals of $A$ with the Jacobson topology and if each member of $J_{0}$ is $\alpha$-invariant, then each member of $J$ is $\alpha$-invariant, and

$$
\sup _{\mathscr{J} \in J_{0}}\left|\sigma\left(\alpha^{\mathscr{J}}\right)\right|=\sup _{\mathscr{J} \in J}\left|\sigma\left(\alpha^{\mathscr{J}}\right)\right|,
$$

provided that the supremum over $J_{0}$ is finite. That each member of $J$ is $\alpha$-invariant follows immediately from the fact that each $\alpha_{t}$ determines a homeomorphism of $J$ 
onto itself with a dense set of fixed points. Suppose that the largest number of elements in any $\sigma\left(\alpha^{\mathscr{I}}\right)$ with $\mathscr{J} \in J_{0}$ is finite, and denote this number by $n$. By lemma 3.3 (which holds just as well for the space of prime closed two-sided ideals as for the space of primitive ideals) for any closed subset $F \subset \mathbb{R}$, the set of $\mathscr{J} \in J$ with $\sigma\left(\alpha^{\mathscr{I}}\right) \subset F$ is closed. Fix $\mathscr{J} \in J$, and choose a net $\left(\mathscr{J}_{\ell}\right)$ in $J_{0}$ converging to $\mathscr{J}$. Passing to a subnet at most $n$ times, we may assume that $\sigma\left(\mathscr{F}_{k}\right)$ converges to a closed set $F \subset \mathbb{R} \cup\{ \pm \infty\}$ with at most $n$ elements. It follows that $\sigma(\mathscr{J})$ is contained in any closed neighbourhood of $F$, and hence that $\sigma(\mathscr{I})$ is contained in $F$; in particular, $|\boldsymbol{\sigma}(\Phi)| \leq n$.

Now, for each $i \in I$, denote by $J_{i}$ the subset of $J$ of prime ideals containing $I_{i}$. By [5, theorem 3.5(iii)] applied to $A / \mathscr{I}_{i}$, each element of $J_{i}$ is $\alpha$-invariant. Moreover, the intersection of the members of $J_{i}$ (even of just the primitive ideals in $J_{i}$ ) is equal to $\mathscr{I}_{i}$. Hence the intersection of the members of $\bigcup_{i \in I} J_{i}$ is $\bigcap_{i \in I} \mathscr{I}_{i}=\{0\}$; in other words, $J_{0}=\bigcup_{i \in I} J_{i}$ is dense in $J$. Since $\sigma\left(\alpha^{\mathscr{I}}\right) \subset \sigma\left(\alpha^{\Phi}\right)$ if $\mathscr{J} \supset \mathscr{I}_{i}$, we have

$$
\sup _{\mathscr{I} \in J_{0}}\left|\sigma\left(\alpha^{\mathscr{J}}\right)\right| \leq \sup _{i \in I}\left|\sigma\left(\alpha^{\mathscr{\Phi}} i\right)\right|
$$

By the preceding paragraph, the desired inequality, which is stronger than (iv), follows.

A second application of the statement in the first sentence of the proof of (iii) $\Rightarrow$ (iv), with $J_{0} \subset J$ the set of primitive ideals of $A$, is that

$$
\sup _{\mathscr{I} \in \operatorname{Prim}(A)}\left|\sigma\left(\alpha^{\mathscr{I}}\right)\right|=\sup _{\mathscr{I} \text { prime }}\left|\sigma\left(\alpha^{\mathscr{I}}\right)\right| \text {. }
$$

(iv) $\Rightarrow$ (ii) and the inequality $\sup _{0 \leq a<c}\left\|P_{a}\right\| \leq \sup _{\mathscr{g} \in \operatorname{Prim}(A)}\left|\sigma\left(\alpha^{\mathscr{I}}\right)\right|$ :

First we note that by $\left[5\right.$, theorem 3.5], $\sigma\left(\alpha_{-i}\right) \neq \mathbb{C}$, so by [5, cor. 2.5] every closed two-sided ideal of $\boldsymbol{A}$ is $\alpha$-invariant. We prove that there exists a closed two-sided ideal $\mathscr{I}_{1}$ of $A$ with Prim $\left(\mathscr{I}_{1}\right)$ dense in Prim $(A)$, and a projection $P_{1} \in B\left(\mathscr{I}_{1}\right)$ such that: image of $P_{1}=\left(\mathscr{I}_{1}\right)^{\alpha \mid \Phi_{1}}((-\infty, 0])$;

kernel of $P_{1}=\left(\mathscr{\Phi}_{1}\right)^{\alpha \mid \Phi_{1}}((0,+\infty))$;

$P_{1}\left(\alpha \mid \mathscr{I}_{1}\right)_{t}=\left(\alpha \mid \mathscr{I}_{1}\right)_{t} P_{1}, \quad t \in \mathbb{R} ;$ and

$\left\|P_{1}\right\| \leq \sup _{J \in \operatorname{Prim}(A)}\left|\sigma\left(\alpha^{\prime}\right)\right|$.

This suffices, because then, using transfinite induction, we get easily (ii) and the desired estimation for $\sup _{0 \leq a<c}\left\|P_{a}\right\|$.

Let us write for each $n \geq 1$

$$
\begin{aligned}
& F_{n}=\{0\} \cup\left\{\lambda \in \mathbb{R} ; \frac{1}{n} \leq|\lambda| \leq n\right\} \subset \mathbb{R}, \\
& \mathscr{F}_{n}=\left\{\mathscr{J} \in \operatorname{Prim}(A) ; \sigma\left(\alpha^{\mathscr{J}}\right) \subset F_{n}\right\} .
\end{aligned}
$$

By lemma 3.3 the $\mathscr{F}_{n}$ 's are closed subsets of Prim $(A)$, and by (iv) $\operatorname{Prim}(A)=$ $\bigcup_{n \geq 1} \mathscr{F}_{n}$. Since Prim $(A)$ is a Baire space, $\mathscr{G}=\bigcup_{n \geq 1}$ (interior of $\mathscr{F}_{n}$ ) is a dense open subset of Prim $(A)$. Hence there exists a closed two-sided ideal $\mathscr{I}_{1}$ of $A$ with

$$
\operatorname{Prim}\left(\mathscr{I}_{1}\right)=\left\{\mathscr{J} \in \operatorname{Prim}(\boldsymbol{A}) ; \mathscr{I}_{1} \not \subset \mathscr{J}\right\}=\mathscr{G}
$$

dense in Prim $(\boldsymbol{A})$. 
For each $n \geq 1$, letting $\mathscr{I}_{1, n}$ denote the closed two-sided ideal of $\boldsymbol{A}$ with $\operatorname{Prim}\left(\mathscr{I}_{1, n}\right)=\left\{\mathscr{J} \in \operatorname{Prim}(A) ; \mathscr{I}_{1, n} \not \subset \mathscr{J}\right\}=$ interior of $\mathscr{F}_{n}$,

we have

$$
\begin{aligned}
& \sigma\left(\alpha^{\Phi_{1, n}}\right) \subset F_{n}, \quad n \geq 1, \\
& \Phi_{1}=\overline{\bigcup_{n \geq 1} \Phi_{1, n}}
\end{aligned}
$$

It follows that

$$
\mathscr{I}_{1, n}=\left(\mathscr{I}_{1, n}\right)^{\alpha \mid \mathscr{I}_{1, n}}((-\infty, 0])+\left(\mathscr{I}_{1, n}\right)^{\alpha \mid \mathscr{S}_{1, n}}((0,+\infty)), \quad n \geq 1 ;
$$

consequently,

$$
\Phi_{1}=\overline{\left(\Phi_{1}\right)^{\alpha \mid \Phi_{1}}((-\infty, 0])+\left(\mathscr{\Phi}_{1}\right)^{\alpha \mid \Phi_{1}}((0,+\infty))}
$$

On the other hand, by corollary 1.2 we have for every $x \in\left(\mathscr{I}_{1}\right)^{\alpha \mid \mathscr{S}_{1}}((-\infty, 0])$ and $y \in\left(\mathscr{\Phi}_{1}\right)^{\alpha \mid \mathscr{J}_{1}}((0,+\infty))$,

$$
\begin{gathered}
\left\|x_{\mathscr{I}}\right\| \leq\left|\sigma\left(\alpha^{\mathscr{I}}\right)\right|\left\|(x+y)_{\mathscr{D}}\right\| \leq\left|\sigma\left(\alpha^{\mathscr{I}}\right)\right|\|x+y\|, \quad \mathscr{J} \in \operatorname{Prim}(A), \\
\|x\|=\sup _{\mathscr{I} \in \operatorname{Prim}(A)}\left\|x_{\mathscr{J}}\right\| \leq\left(\sup _{\mathscr{I} \in \operatorname{Prim}(A)}\left|\sigma\left(\alpha^{\mathscr{I}}\right)\right|\right)\|x+y\|,
\end{gathered}
$$

where $a_{\mathscr{F}}$ denotes the canonical image of $a \in A$ in $A / \mathscr{J}$.

We conclude that there exists a projection $P_{1} \in B\left(\Phi_{1}\right)$ of norm $\leq$ $\sup _{\mathscr{J} \in \operatorname{Prim}(A)}\left|\sigma\left(\alpha^{\mathscr{I}}\right)\right|$ such that:

image of $P_{1}=\left(\mathscr{I}_{1}\right)^{\alpha \mid \mathscr{S}_{1}}((-\infty, 0])$;

kernel of $P_{1}=\left(\mathscr{I}_{1}\right)^{\alpha \mid \mathscr{G}_{1}}((0,+\infty))$.

Since $\left(\alpha \mid \Phi_{1}\right)_{t} P_{1}\left(\alpha \mid \mathscr{I}_{1}\right)_{-t}$ is, for each $t \in \mathbb{R}$, a linear projection with the same image and kernel as $P_{1}$, we have automatically

$$
P_{1}\left(\alpha \mid \mathscr{I}_{1}\right),=\left(\alpha \mid \mathscr{I}_{1}\right), P_{1}, \quad t \in \mathbb{R} .
$$

(ii) $\Rightarrow(\mathrm{v})$ :

From (i) $\Rightarrow$ (iv) established above, applied for each $a, 0 \leq a<c$, we see that the $C^{*}$-dynamical system $\left(\mathscr{\Phi}_{a+1} / \mathscr{I}_{a}, \alpha^{\Phi_{a}} \mid \mathscr{I}_{a+1}\right)$ satisfies the equivalent conditions of [5, theorem 3.5 and cor. 4.5]. It follows in particular that $t \mapsto \alpha_{t}^{* *}$ is a $\sigma\left(A^{* *}, A^{*}\right)$ continuous one-parameter group of automorphisms of $A^{* *}$ - denote it by $\alpha^{* *}$. From (ii) it follows immediately that the $W^{*}$-dynamical system $\left(A^{* *}, \alpha^{* *}\right)$ satisfies condition (iii) of theorem 3.1 in the special case $F=(-\infty, 0]$; in particular, it satisfies condition (i) of theorem 3.1 (and therefore it satisfies all the equivalent conditions of theorem 3.1). Moreover, since $P_{(-\infty, 0]}^{\alpha *}$ is the direct sum of all $P_{a}^{* *}$, we have

$$
\sup _{0 \leq a<c}\left\|P_{a}\right\|=\left\|P_{(-\infty, 0]}^{\alpha * *}\right\| \text {. }
$$

(v) $\Rightarrow(\mathrm{i})$ :

It follows from condition (iv) of theorem 3.1 that for each irreducible representation $\pi$ of $A$ the restriction $\left(\alpha^{* *}\right)^{\pi}$ of $\alpha^{* *}$ to the weak closure of $\pi(A)$ has finite spectrum, and that the supremum of these cardinalities is finite. Since $\sigma\left(\alpha^{\text {ker }}\right) \subseteq$ $\sigma\left(\left(\alpha^{* *}\right)^{\pi}\right)$, we have condition (iii) of the present theorem, with the family $\left(\Phi_{i}\right)$ taken to be Prim $A$. As shown above, condition (iii) implies condition (ii), and condition (i) follows trivially. 
Finally, the implication (v) $\Rightarrow$ (vi) is trivial, and the implication (vi) $\Rightarrow(v)$ follows easily by [18, cor. 2.5$]$, [5, prop. 2.2], and the uniform boundedness principle.

(3.5) Corollary. Let $(A, \alpha)$ be a one-parameter $C^{*}$-dynamical system.

If $B$ is an $\alpha$-invariant $C^{*}$-subalgebra of $A$ and $(A, \alpha)$ satisfies the equivalent conditions of theorem 3.4, then $(B, \alpha \mid B)$ also satisfies them.

If $\mathscr{I}$ is a closed two-sided ideal of $A$, then $(A, \alpha)$ satisfies the equivalent conditions of theorem 3.4 if and only if $\mathscr{I}$ is $\alpha$-invariant and $(\mathscr{I}, \alpha \mid \mathscr{I})$ and $\left(A / \mathscr{I}, \alpha^{\mathscr{J}}\right)$ satisfy them. Proof. The first statement follows from corollary 3.2 by making use of condition (v) from theorem 3.4. Also the second statement follows easily by using condition (v) from theorem 3.4.

Unfortunately, if a one-parameter $C^{*}$-dynamical system $(A, \alpha)$ satisfies the equivalent conditions from theorem 3.4 , there does not necessarily exist a projection $P_{(-\infty, 0]}^{\alpha} \in B(A)$ with:

image of $P_{(-\infty, 0]}^{\alpha}=A^{\alpha}((-\infty, 0])$;

kernel of $P_{(-\infty, 0]}^{\alpha}=A^{\alpha}((0,+\infty))$.

Indeed let $A$ be the $C^{*}$-algebra of all convergent sequences of complex $2 \times 2$ matrices, $a$ the self-adjoint element of $\boldsymbol{A}$ corresponding to the sequence

$$
\left(\begin{array}{ll}
0 & 0 \\
0 & 1
\end{array}\right),\left(\begin{array}{cc}
0 & 0 \\
0 & 1 / 2
\end{array}\right), \ldots,\left(\begin{array}{cc}
0 & 0 \\
0 & 1 / n
\end{array}\right), \ldots,
$$

and $\alpha$ the uniformly continuous one-parameter group of *-automorphisms of $\boldsymbol{A}$ defined by

$$
\alpha_{t}(x)=\exp (i t a) x \exp (-i t a) .
$$

It is easy to see that $(A, \alpha)$ satisfies condition (iv) from theorem 3.4 , but

$$
\begin{aligned}
A^{\alpha}((-\infty, 0])= & \text { the set of all convergent sequences of } \\
& \text { matrices of the form }\left(\begin{array}{ll}
e & f \\
0 & h
\end{array}\right), \\
A^{\alpha}((0,-\infty))= & \text { the set of all sequences of matrices of } \\
& \text { the form }\left(\begin{array}{ll}
0 & 0 \\
g & 0
\end{array}\right) \text {, which converge to }\left(\begin{array}{ll}
0 & 0 \\
0 & 0
\end{array}\right),
\end{aligned}
$$

so

$$
A \neq \overline{A^{\alpha}((-\infty, 0])+A^{\alpha}((0,+\infty))} \text {. }
$$

The explanation of the above deviation from the case of $W^{*}$-dynamical systems relies on the fact that all one-parameter $W^{*}$-dynamical systems satisfy the weak ergodic property from $[16, \S 3]$, whilst for general one-parameter $C^{*}$-dynamical systems this is not true. More concretely, the following second $C^{*}$-algebra counterpart to theorem 3.1 holds:

(3.6) Theorem. Let $(A, \alpha)$ be a one-parameter $C^{*}$-dynamical system. Then the following statements are equivalent: 
(i) there exists a projection $P_{(-\infty, 0]}^{\alpha} \in B(A)$ with:

image of $P_{(-\infty, 0]}^{\alpha}=A^{\alpha}((-\infty, 0])$,

kernel of $P_{(-\infty, 0]}^{\alpha}=A^{\alpha}((0,+\infty))$,

$P_{(-\infty, 0]}^{\alpha} \alpha_{t}=\alpha_{t} P_{(-\infty, 0]}^{\alpha}, \quad t \in \mathbb{R}$;

(ii) $\alpha$ has the Hilbert transform property in every $x \in A$; that is, the limit

$$
H^{\alpha}(x)=\frac{i}{\pi} \sigma\left(A, A^{*}\right)-\lim _{\substack{0<\varepsilon \rightarrow 0 \\ \delta \rightarrow+\infty}} \int_{\varepsilon \leq|t|<\delta} \frac{1}{t} \alpha_{t}(x) d t \in A
$$

exists for each $x \in A$;

(iii) $(A, \alpha)$ satisfies the equivalent conditions from theorem 3.4 and

$$
\left(\bigcup_{K \in(-\infty, 0) \text { compact }} A^{\alpha}(K)\right)+A^{\alpha}(\{0\})+\left(\bigcup_{K \subset(0,+\infty) \text { compact }} A^{\alpha}(K)\right)
$$

is dense in $A$, or, equivalently,

$$
\underset{\delta \rightarrow+\infty}{\operatorname{norm}-\lim } \frac{1}{2 \delta} \int_{-\delta}^{\delta} \alpha_{t}(x) d t
$$

exists for all $x \in A$.

Proof. We note that if (i) holds, then the formula

$$
P_{[0,+\infty)}^{\alpha}(x)=\left(P_{(-\infty, 0]}^{\alpha}\left(x^{*}\right)\right)^{*}
$$

defines a projection $P_{[0,+\infty)}^{\alpha} \in B(A)$ with:

$$
\begin{aligned}
& \text { image of } P_{[0,+\infty)}^{\alpha}=A^{\alpha}([0,+\infty)) ; \\
& \text { kernel of } P_{[0,+\infty)}^{\alpha}=A^{\alpha}((-\infty, 0)) \text {. }
\end{aligned}
$$

Using this remark, (i) $\Leftrightarrow$ (ii) follows from [18, cor. 3.6]. We note also, that by [16, cor. 3.5],

$$
\left(\bigcup_{K \subset(-\infty, 0) \text { compact }} A^{\alpha}(K)\right)+A^{\alpha}(\{0\})+\left(\bigcup_{K \subset(0,+\infty) \text { compact }} A^{\alpha}(K)\right)
$$

is dense in $A$ if and only if

$$
\underset{\delta \rightarrow+\infty}{\operatorname{norm}-\lim } \frac{1}{2 \delta} \int_{-\delta}^{\delta} \alpha_{t}(x) d t
$$

exists for all $x \in A$. The equivalence of (iii) with (i) and (ii) is an immediate consequence of $[18$, cor. 3.7$]$.

We remark that, contrary to corollary 3.5 , if $(A, \alpha)$ is a one-parameter $C^{*}$-dynamical system, $\mathscr{I}$ an $\alpha$-invariant closed two-sided ideal of $A$, and $\alpha \mid \mathscr{I}$ and $\alpha^{\mathscr{F}}$ satisfy the equivalent conditions from theorem 3.6, then this does not necessarily hold for $\alpha$ : we can take, for example, $(A, \alpha)$ as in the example before theorem 3.6 and $\mathscr{I}=$ the set of all complex $2 \times 2$ matrices, which converge to

$$
\left(\begin{array}{ll}
0 & 0 \\
0 & 0
\end{array}\right)
$$

During the writing of this paper L. Zsidó was visiting IHES, Bures-sur-Yvette. G. A. Elliott was a Science and Engineering Research Council Senior Visiting Fellow at the University of Warwick. 


\section{REFERENCES}

[1] W. Arveson. On groups of automorphisms of operator algebras. J. Funct. Anal. 15 (1974), 217-243.

[2] I. Ciorănescu \& L. Zsidó. Analytic generators for one-parameter groups. Tôhoku Math. J. 28 (1976), 327-362.

[3] I. Ciorănescu \& L. Zsidó. On spectral subspaces of some unbounded groups of operators. Rev. Roumaine Math. Pures Appl. 21 (1976), 817-850.

[4] F. Combes \& C. Delaroche. Représentations des groupes localement compacts et applications aux algèbres d'opérateurs. Astérisque 55 (1978).

[5] G. A. Elliott \& L. Zsidó. Almost uniformly continuous automorphism groups of operator algebras. J. Operator Theory 8 (1982), 227-277.

[6] J. G. Glimm. A Stone-Weierstrass theorem for $C^{*}$-algebras, Ann. of Math. 72 (1960), 216-244.

[7] F. P. Greenleaf. Invariant Means on Topological Groups and their Applications. Van NostrandReinhold: New York, 1969.

[8] H. Halpern. Irreducible module homomorphisms of a von Neumann algebra into its center. Trans. Amer. Math. Soc. 140 (1969), 195-221.

[9] E. Hille \& R. S. Phillips. Functional Analysis and Semigroups. American Mathematical Society: Providence, Rhode Island, 1957.

[10] M. J. Kadets \& B. S. Mityagin. Complemented subspaces in Banach spaces. Uspekhi Mat. Nauk 28, No. 6 (1973), 77-94.

[11] D. Olesen. On spectral subspaces and their applications to automorphism groups. In Symposia Mathematica Vol. XX, pp. 253-296. Academic Press: London, 1976.

[12] G. K. Pedersen. $C^{*}$-algebras and their Automorphism Groups. Academic Press: London, 1979.

[13] F. Riesz \& B. Sz.-Nagy. Lecons d'Analyse Fonctionelle, $3^{e}$ édition. Akadémiai Kiadó: Budapest, 1955.

[14] W. Rudin. Projection on invariant subspaces. Proc. Amer. Math. Soc. 13 (1962), 429-432.

[15] S. Sakai. $C^{*}$-algebras and $W^{*}$-algebras. Springer-Verlag: New York, 1971.

[16] L. Zsidó. Spectral and ergodic properties of the analytic generator. J. Approx. Theory 20 (1977), $77-138$.

[17] L. Zsidó. On spectral subspaces associated to locally compact abelian groups. Advances in Math. 36 (1980), 213-276.

[18] L. Zsidó. Spectral properties of the analytic generator and singular integrals. Atti Accad. Naz. Lincei Mem. Cl. Sci. Fis. Mat. Natur. Sez. Ia (8) 17 (1983), 105-134. 